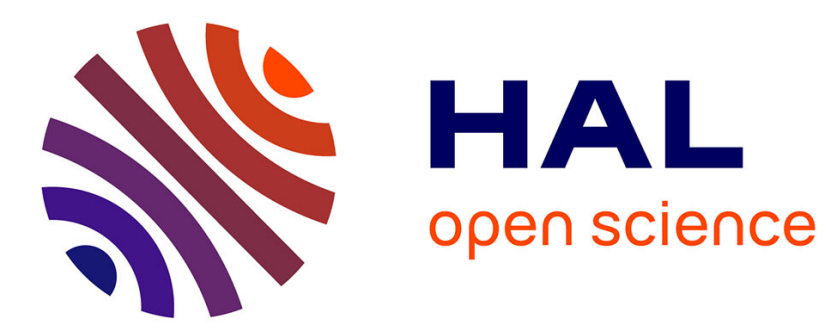

\title{
Capillary jet breakup by noise amplification
}

Stéphane Le Dizès, Emmanuel Villermaux

\section{- To cite this version:}

Stéphane Le Dizès, Emmanuel Villermaux. Capillary jet breakup by noise amplification. Journal of Fluid Mechanics, 2017, 810, pp.281-306. hal-01430563

\section{HAL Id: hal-01430563 https://hal.science/hal-01430563}

Submitted on 12 Apr 2018

HAL is a multi-disciplinary open access archive for the deposit and dissemination of scientific research documents, whether they are published or not. The documents may come from teaching and research institutions in France or abroad, or from public or private research centers.
L'archive ouverte pluridisciplinaire HAL, est destinée au dépôt et à la diffusion de documents scientifiques de niveau recherche, publiés ou non, émanant des établissements d'enseignement et de recherche français ou étrangers, des laboratoires publics ou privés. 


\title{
Capillary jet breakup by noise amplification
}

\author{
S. Le Dizès ${ }^{1}$ \& E. Villermaux ${ }^{1,2}$ \\ ${ }^{1}$ Aix Marseille Univ, CNRS, Cent Marseille, IRPHE, Marseille, France \\ ${ }^{2}$ Institut Universitaire de France, Paris, France
}

(Received 28 October 2016)

\begin{abstract}
A liquid jet falling by gravity ultimately destabilizes by capillary forces. Accelerating as it falls, the jet thins and stretches, causing the capillary instability to develop on a spatially varying substrate. We discuss quantitatively the interplay between instability growth, jet thinning and longitudinal stretching for two kinds of perturbations, either solely introduced at the jet nozzle exit, or affecting the jet all along its length. The analysis is conducted for any values of the liquid properties for sufficiently large flow rate. In all cases, we determine the net gain of the most dangerous perturbation for all downstream distances, thus predicting the jet length, the wavelength at breakup and the resulting droplet size.
\end{abstract}

\section{Introduction}

Seemingly simple questions are not always the simplest to answer quantitatively. A canonical illustration of this affirmation is the apparently simple problem of a liquid thread, falling from a nozzle by it own weight under the action of gravity, as shown in figure 1. As it falls, the thread eventually fragments into drops, a fact that we understand because it has locally a columnar shape, and thus suffers a capillary instability. But how far from the nozzle exit does breakup happen? Even a distracted look at the possible scenarii lets one glimpse the potential difficulties of a precise analysis: a distance $z$ is the product of a velocity $u$ by a time $\tau$

$$
z=u \tau \text {. }
$$

Capillary breakup occurs within a time $\tau$ which depends on the thread radius $h$, on the liquid density $\rho$, viscosity $\eta$ and surface tension $\gamma$, and we know that most of this time is spent at developing an instability about the quasi-columnar shape of the thread, the subsequent phenomena occurring around the pinching instant at the drops separation being comparatively much faster (Eggers \& Villermaux 2008). The time $\tau$ is either the capillary time $\sqrt{\rho h^{3} / \gamma}$ when inertia and surface tension are solely at play, or the viscous capillary time $\eta h / \gamma$ if viscous effects dominantly slow down the unstable dynamics. When the jet issues from the nozzle ballistically, keeping its velocity and radius constant, the problem is indeed simple, and amounts to estimate correctly the relevant timescale $\tau$ to compute the so-called 'Liquid intact length' of the jet (see the corresponding section in Eggers \& Villermaux (2008) for a complete discussion and experimental references, including the case when the jet suffers a shear instability with the surrounding environment). Subtleties arise when the axial velocity of the jet depends on axial distance $z$.

A jet falling in the direction of gravity accelerates. If fed at a constant flow rate at the nozzle, stationarity implies that the thread radius thins with increasing distances from the exit. Therefore, if both $u$ and $h$ depend on downstream distance, which estimates will correctly represent the breakup distance $z$ in equation (1.1)? Those at the nozzle exit, those at the breakup distance, or a mixture of the two? As the radius thins, the instability 


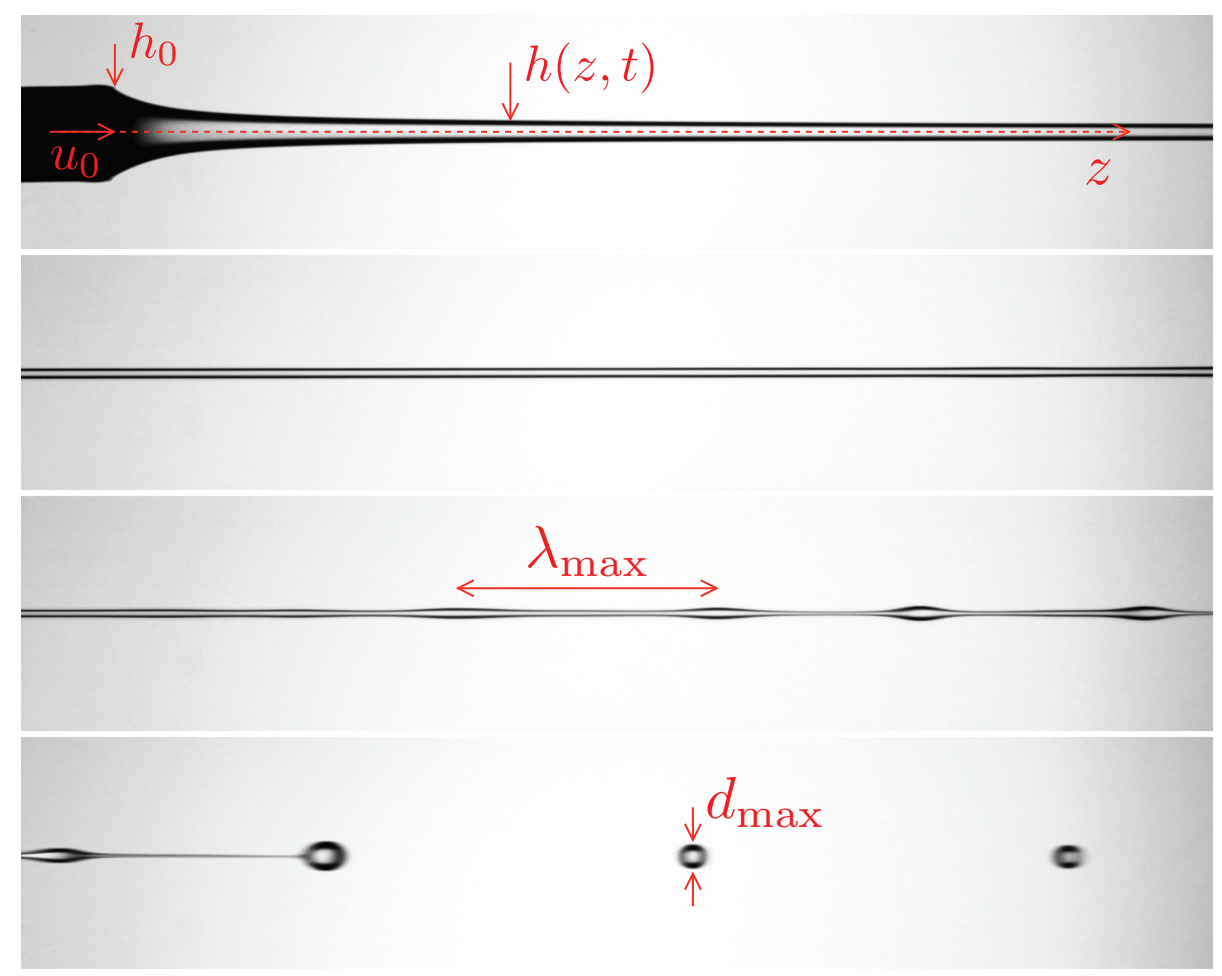

Figure 1: Four successive panels showing a liquid jet (density $950 \mathrm{~kg} / \mathrm{m}^{3}$, viscosity $\eta=$ $50 \times 10^{-3} \mathrm{~Pa} \mathrm{~s}$ ) issuing from a round tube with radius $h_{0}=2 \mathrm{~mm}$ at velocity $u_{0}=1 \mathrm{~cm} / \mathrm{s}$, stretching in the gravity field (aligned with the $z$ direction), and thinning as it destabilizes through the growth of bulges separated by $\lambda_{\max }$ at breakup, producing stable drops of diameter $d_{\max }$.

may switch from an inertia to a viscous dominated régime. Then, which timescale $\tau$ should be considered to compute $z$ ?

The detailed problem is even more subtle: The capillary instability amplifies preferentially a varicose perturbation, adjacent bulges along the thread feeding on the thinner ligament linking them (figure 1). The most amplified wavelength is proportional to $h$, the other wavelengths having a weaker growth rate. Since the jet accelerates, mass conservation of the incompressible liquid also implies that the distance between two adjacent instability crests increases with larger distances from the nozzle exit. The capillary instability has thus to compete with another phenomenon, namely jet stretching, characterized by another timescale $\left(\partial_{z} u\right)^{-1}$. There are thus three timescales which may potentially contribute to $\tau$, and which all depend intrinsically on the distance to the nozzle. Deciding a-priori which one will dominate and how is a hazardous exercise.

Deciphering the relative importance of the coupled effects mentioned above requires an instability analysis accounting for both the substrate deformation (jet stretching), and for the modification of the local instability dispersion relation as the jet thins (to describe the growing relative influence of viscosity). That question has been envisaged in the very viscous limit by Tomotika (1936), for the particular case where $u$ increases linearly with $z$ by Frankel \& Weihs (1985, 1987), and more recently by Senchenko \& Bohr (2005), Sauter \& Buggisch (2005) and Javadi et al. (2013) for a gravitationally accelerated jet.

These last authors quantified the maximum gain that perturbations can reach at a 
given location using a local plane wave decomposition (WKBJ approximation). By choosing adequately the gain needed for breakup, they were able to collapse measurements of the breakup distance on a theoretical curve. They also obtained an asymptotic expression in the viscous regime consistent with the anticipated scaling law which compares the viscous capillary timescale based on the current jet radius to the stretching time of the jet.

In the present work, we use a similar approach as Javadi et al. (2013) by searching maximum perturbation gains using WKBJ approximations. In addition to providing much more details, we extend their analysis in several ways. We first consider all the regimes ranging from very viscous to inviscid. We then compare the maximum gain and the most dangerous frequency of the perturbations for two types of excitation: (1) nozzle excitation (the perturbation is introduced at the nozzle only); (2) background noise (the perturbation is present everywhere). We finally provide predictions for the breakup wavelength and the resulting droplet size.

The paper is organized as follows: In $\S 2$, we present the mathematical formulation by providing the model for the base flow and the perturbations. An expression of the perturbation gain is derived using the WKBJ framework. In $\S 3$, the result of the optimization procedure maximizing the gain is provided for each type of excitation. The break up distance, the most dangerous frequency, the wavelength and the droplet size are analysed as functions of the gain and fluid viscosity (Ohnesorge number Oh). Asymptotic formulas for weak and strong viscosity (small and large $\mathrm{Oh}$ ) are provided in this section, though their derivation is moved in an appendix at the end of the paper. For nozzle excitation, a peculiar behavior of the optimal perturbation observed for intermediate Ohnesorge numbers $(0.1<\mathrm{Oh}<1)$ is further discussed in $\S 4$. We show that the peak of the breakup wavelength obtained for $\mathrm{Oh} \approx 0.3$ is related to a property of the local dispersion relation outside the instability band. The results are compared to local predictions in $\S 5$ and applied to realistic configurations in $\S 6$.

\section{Mathematical formulation}

We consider an axisymmetric liquid jet falling vertically by the action of gravity $g$. The jet has a radius $h_{0}$ and a characteristic velocity $u_{0}$ at the nozzle (figure 1 ). The fluid has a density $\rho$, a viscosity $\nu=\eta / \rho$, and a surface tension $\gamma$. The surrounding environment is considered as evanescent, and is neglected.

\subsection{Base Flow}

Spatial and time variables are non-dimensionalized using the radius $h_{0}$, and the capillary time $\tau_{c}=\sqrt{\rho h_{0}^{3} / \gamma}$ respectively. The base flow is governed by three parameters

$$
\begin{array}{r}
Q=u_{0} \sqrt{\frac{\rho h_{0}}{\gamma}}, \text { The flow rate, } \\
\mathrm{Oh}=\nu \sqrt{\frac{\rho}{\gamma h_{0}}}, \text { The Ohnesorge number, } \\
\text { Bo }=\frac{\rho g h_{0}^{2}}{\gamma} \text { The Bond number. }
\end{array}
$$

One could alternatively use the Weber number $\mathrm{We}=Q^{2}$ instead of the dimensionless flow rate. The Ohnesorge number is the ratio of the viscous capillary timescale to the capillary timescale. We describe the liquid jet by the one-dimensional model (Trouton 
1906; Weber 1931; Eggers \& Villermaux 2008)

$$
\begin{aligned}
\frac{\partial A}{\partial t}+\frac{\partial(A u)}{\partial z} & =0 \\
\frac{\partial u}{\partial t}+u \frac{\partial u}{\partial z} & =3 \mathrm{Oh} \frac{1}{A} \frac{\partial}{\partial z}\left(A \frac{\partial u}{\partial z}\right)+\frac{\partial \mathrm{K}}{\partial z}+\mathrm{Bo}
\end{aligned}
$$

with

$$
\mathrm{K}=\frac{4 A A_{\mathrm{zz}}-2 A_{z}^{2}}{\left[4 A+A_{z}^{2}\right]^{3 / 2}}-\frac{2}{\left[4 A+A_{z}^{2}\right]^{1 / 2}}
$$

where $u(z, t)$ is the local axial velocity, $A=h^{2}$ is the square of the local radius $h(z, t), z$ is the axial coordinate oriented downward, $t$ is the time variable, $A_{z}$ and $A_{\mathrm{zz}}$ are respectively, the first and second derivative of $A$ with respect to $z$. The boundary conditions at the nozzle are

$$
A(z=0, t)=1, u(z=0, t)=Q
$$

The stationary base flow satisfies

$$
\begin{array}{r}
\frac{\partial\left(A_{0} U_{0}\right)}{\partial z}=0 \\
U_{0} \frac{\partial U_{0}}{\partial z}=3 \mathrm{Oh} \frac{1}{A_{0}} \frac{\partial}{\partial z}\left(A_{0} \frac{\partial U_{0}}{\partial z}\right)+\frac{\partial \mathrm{K}_{0}}{\partial z}+\text { Bo } .
\end{array}
$$

The first equation gives

$$
A_{0} U_{0}=Q
$$

We will consider the régime where the jet base flow is inertial and given at leading order by

$$
U_{0} \frac{\partial U_{0}}{\partial z}=\text { Bo. }
$$

This hypothesis amounts to neglect viscous and curvature effects in the jet evolution. Because it accelerates as it falls, the jet gets thinner and slender. Curvature effects along $z$ thus soon vanish (unless the jet is initially very small, see Rubio-Rubio et al. 2013), and viscous stresses applying on the jet cross section are also soon overcomed by the gravity force (beyond a physical distance from the nozzle of order $\sqrt{\nu u_{0} / g}$, see Clarke 1969). Equations (2.6) and (2.7) thus give

$$
\begin{aligned}
& U_{0}(z)=\sqrt{2 \operatorname{Bo} z+Q^{2}}, \\
& A_{0}(z)=\frac{Q}{\sqrt{2 \operatorname{Bo} z+Q^{2}}} .
\end{aligned}
$$

Plugging these expressions in the viscous and curvature terms of equation (2.2b), one observe that they are both decreasing with $z$. Viscous and curvature terms are therefore negligible along the entire jet, if they are already negligible in the vicinity of the nozzle exit. This is satisfied if the flow rate is sufficiently large, and more precisely if the following conditions are met

$$
\begin{array}{r}
Q \gg 1, \\
Q^{2} \gg \text { Bo, } \\
Q^{3} \gg \text { Bo Oh. }
\end{array}
$$

Note that if the parameters $Q$, Bo and Oh are defined from the local values of $U_{0}$ and $A_{0}$, conditions (2.9a-c) are always satisfied sufficiently far away from the nozzle (e.g. Sauter 
\& Buggisch 2005). Since the phenomena we will describe result from a dynamics which integrates over distances much larger than the jet initial radius, we use here (2.8) as a good approximation of the base flow everywhere.

For simplicity, we assume in the sequel that $Q$ is the only large parameter, Bo and $\mathrm{Oh}$ being of order 1 or smaller. Both $U_{0}$ and $A_{0}$ then vary with respect to the slow variable

$$
Z=\frac{z}{z_{o}}+1,
$$

as

$$
\begin{aligned}
& U_{0}(Z)=Q \sqrt{Z} \\
& A_{0}(Z)=1 / \sqrt{Z}
\end{aligned}
$$

where

$$
z_{o}=\frac{Q^{2}}{2 \mathrm{Bo}}
$$

is the (large) nondimensionalized variation scale of the base flow.

\subsection{Perturbations}

We now consider linear perturbations $\left(u_{p}, A_{p}\right)$ in velocity and cross-section to the above base flow. These perturbations satisfy the linear system

$$
\begin{aligned}
\frac{\partial A_{p}}{\partial t} & =-\frac{\partial\left(A_{p} U_{0}+A_{0} u_{p}\right)}{\partial z} \\
\frac{\partial u_{p}}{\partial t}+\frac{\partial u_{p} U_{0}}{\partial z} & =3 \frac{\mathrm{Oh}}{A_{0}}\left\{\frac{\partial}{\partial z}\left(A_{0} \frac{\partial u_{p}}{\partial z}+A_{p} \frac{\partial U_{0}}{\partial z}\right)-\frac{A_{p}}{A_{0}} \frac{\partial}{\partial z}\left(A_{0} \frac{\partial U_{0}}{\partial z}\right)\right\}+\frac{\partial \mathcal{L}\left(A_{p}\right)}{\partial z}
\end{aligned}
$$

where $\mathcal{L}\left(A_{p}\right)$ is the linear operator obtained by linearizing $K-K_{0}$ around $A_{0}$.

We want to analyze these perturbations in the 'jetting' regime when the jet is globally stable. More precisely, we do not consider the global transition that leads to dripping and which has been studied elsewhere (Le Dizès 1997; Sauter \& Buggisch 2005; Rubio-Rubio et al. 2013). We are interested in the growth of the perturbations that give rise to the formation of droplets far away from the nozzle. In this regime, the jet is convectively unstable: the perturbations are advected downstream as they grow. We expect droplets to form when the perturbation has reached a sufficiently large amplitude. Of particular interest is the maximum amplitude that perturbations can reach at a given location $z_{f}$ from a fixed level of noise. This amounts to calculate the maximum spatial gain that perturbations can exhibit at a given downstream location. For this purpose, we will consider two situations:

(a) Fluctuations are mainly present at the nozzle as in laboratory experiments where the jet nozzle is vibrated for instance (Sauter \& Buggisch 2005). In that case, we are interested in the spatial gain at $z_{f}$ of perturbations generated at the nozzle $z=0$.

(b) The jet is subject to a background noise which acts at every $z$ location. In that case, we are interested in the maximum gain at $z_{f}$ of perturbations which originates from anywhere along the jet. In other words, we are interested in the spatial gain between $z_{i}$ and $z_{f}$, where $z_{i}$ is chosen such that the gain is maximum. Obviously, the gain in that case is larger than in (a), since $z=0$ is one particular excitation location among the many possible in that case.

The base flow is stationary; a temporal excitation at a given location with a fixed frequency leads to a temporal response in the whole jet with the same frequency. As the 
jet can be forced on $A$ or on $u$, we expect two independent spatial structures associated with each frequency. If we write

$$
\left(u_{p}, A_{p}\right)=(\tilde{u}, \tilde{A}) e^{-i \omega t}+c . c .,
$$

the normalized solution forced in $u$ at the nozzle will satisfy $\tilde{A}(z=0)=0, \tilde{u}(z=0)=1$, while the one forced in $A$ at the nozzle will satisfy $\tilde{A}(z=0)=1, \tilde{u}(z=0)=0$. A linear combination of these two solutions can be used to obtain the normalized solution forced in $u$ or forced in $A$ at any location $z_{i}$.

We then define a spatial gain in $A$ from $z_{i}$ to $z_{f}$ from the solution forced in $A$ at $z_{i}$ by $G_{A}\left(z_{i}, z_{f}\right)=\left|\tilde{A}\left(z_{f}\right)\right|$. Similarly, we define a spatial gain in $u$ from $z_{i}$ to $z_{f}$ from the solution forced in $u$ at $z_{i}$ by $G_{u}\left(z_{i}, z_{f}\right)=\left|\tilde{u}\left(z_{f}\right)\right|$.

Both $U_{0}$ and $A_{0}$ depend on the slow spatial variable $Z$. Anticipating that the typical wavelength will be of order 1, a local plane wave approximation (WKBJ approximation) can be used (Bender \& Orszag 1978). In other words, each time-harmonic perturbation amplitude can be written as a sum of expressions of the form (WKBJ approximation)

$$
(\tilde{u}, \tilde{A})=(v(Z), a(Z)) e^{i z_{o} \int^{Z} k(s) d s},
$$

where $k(Z), v(Z)$ and $a(Z)$ depend on the slow variation scale of the base flow. With the WKBJ ansatz, the perturbations equations become at leading order in $1 / z_{o}$

$$
\begin{array}{r}
\left(-i \omega+i k U_{0}\right) a+i k A_{0} v=0, \\
\left(-i \omega+i k U_{0}\right) v=-3 \operatorname{Oh} k^{2} v+\frac{i k}{2 A_{0}^{3 / 2}}\left(1-k^{2} A_{0}\right) a .
\end{array}
$$

These two equations can be simultaneously satisfied (by non-vanishing fields) if and only if

$$
\left(-i \omega+i k U_{0}\right)^{2}+3 \mathrm{Oh} k^{2}\left(-i \omega+i k U_{0}\right)-\frac{k^{2}}{2 \sqrt{A_{0}}}\left(1-k^{2} A_{0}\right)=0 .
$$

This equation provides $k$ as a function of $Z$. Expressions for $v(Z)$ and $a(Z)$ can be obtained by considering the problem to the next order (see appendix B).

Among the four possible solutions to (2.17), only the two wavenumbers corresponding to waves propagating downstream are allowed. As explained in Bers (1983) (see also Huerre \& Monkewitz 1990), these wavenumbers are the analytic continuations for real $\omega$ of functions satisfying $\Im m(k)>0$ for large $\Im m(\omega)$. They are well-defined in the convective regime that we consider here.

If $\omega=\bar{\omega} Q$ with $\bar{\omega}=O(1)$, the wavenumbers associated with the downstream propagating waves can be expanded as

$$
k \sim k_{o}+\frac{k_{1}}{Q}
$$

where $k_{o}$ is found to be identical for both waves:

$$
k_{0}=\frac{\omega}{U_{0}}=\bar{\omega} A_{0} .
$$

At the order $1 / Q$, we get

$$
k_{1}=-i\left\{\frac{k_{0} A_{0}^{3 / 4}}{\sqrt{2}} \sqrt{1-A_{0} k_{0}^{2}+\frac{9 \mathrm{Oh}^{2} \sqrt{A_{0}} k_{0}^{2}}{2}}-\frac{3 \mathrm{Oh} k_{0}^{2} A_{0}}{2}\right\} .
$$

The two wavenumbers are obtained by considering the two possible values of the square root. Although both waves are needed to satisfy the boundary conditions at the nozzle, 
the solution is rapidly dominated downstream by a single wave corresponding to the wavenumber with the smallest imaginary part.

Both the solution forced in $A$ and the solution forced in $u$ are thus expected to have a similar WKBJ approximation (2.15). The main contribution to the two gains $G_{A}\left(z_{i}, z_{f}\right)$ and $G_{u}\left(z_{i}, z_{f}\right)$ is therefore expected to be the same and given by the exponential factor

$$
G\left(z_{i}, z_{f}\right)=e^{S\left(Z_{i}, Z_{f}\right)}
$$

where

$$
S\left(Z_{i}, Z_{f}\right)=-z_{o} \int_{Z_{i}}^{Z_{f}} \Im m(k)(Z) d Z=-\frac{z_{o}}{Q} \int_{Z_{i}}^{Z_{f}} \Im m\left(k_{1}\right)(Z) d Z .
$$

This implicitly assumes that $z_{o} / Q=Q / 2$ Bo is large. When $z_{o} / Q=O(1)$, the WKBJ approach remains valid but the gain (2.21) is of same order of magnitude as the variation of $v$ and $a$. In that case, one should a priori take into account the amplitude $v$ and $a$ provided in Appendix B and apply explicitly the boundary conditions at the forcing location. It leads to different gains for a forcing in velocity and a forcing in radius.

The gain $G$ is associated with the temporal growth of the local perturbation. Indeed, $S$ can be written as

$$
S=z_{o} \int_{Z_{i}}^{Z_{f}} \frac{\sigma\left(k_{l}(Z), \mathrm{Oh}_{l}(Z)\right)}{\tau_{c_{l}}(Z) U_{0}(Z)} d Z
$$

where $\sigma(k, \mathrm{Oh})$ is the growth rate of the capillary instability for the 1D model:

$$
\sigma(k, \mathrm{Oh})=\frac{k}{\sqrt{2}} \sqrt{1-k^{2}+\frac{9 \mathrm{Oh}^{2} k^{2}}{2}}-\frac{3 \mathrm{Oh} k^{2}}{2} .
$$

The local wavenumber $k_{l}(Z)$, local Ohnesorge $\mathrm{Oh}_{l}(Z)$ and local capillary time scale $\tau_{c_{l}}(Z)$ vary as

$$
\begin{aligned}
& k_{l}(Z)=\bar{\omega} Z^{-3 / 8}, \\
& \mathrm{Oh}_{l}(Z)=\mathrm{Oh} Z^{1 / 8}, \\
& \tau_{c_{l}}(Z)=Z^{-3 / 8} .
\end{aligned}
$$

In the following, we write $S$ as

$$
S=\frac{z_{o}}{\sqrt{2} Q} \bar{S}\left(Z_{f}, Z_{i}, \mathrm{Oh}, \bar{\omega}\right)
$$

with

$$
\bar{S}\left(Z_{i}, Z_{f}, \bar{\omega}, \mathrm{Oh}\right)=\bar{\omega} \int_{Z_{i}}^{Z_{f}} z^{-7 / 8}\left(\sqrt{1-\bar{\omega}^{2} z^{-3 / 2}+\frac{9 \mathrm{Oh}^{2} \bar{\omega}^{2}}{2} z^{-5 / 4}}-\frac{3 \mathrm{Oh} \bar{\omega}}{\sqrt{2}} z^{-5 / 8}\right) d z .
$$

Our objective is to find the frequency $\bar{\omega}$ that gives the largest value of $\bar{S}$ at a given $Z_{f}$. For the type of perturbations in case (a) (nozzle excitation), $Z_{i}=1$, and we are looking for

$$
\bar{S}_{\max }^{(a)}\left(Z_{f}, \mathrm{Oh}\right)=\max _{\bar{\omega}} \bar{S}\left(1, Z_{f}, \bar{\omega}, \mathrm{Oh}\right) .
$$

For the type of perturbations in case (b) (background noise), the gain is maximized over all $Z_{i}$ between 1 and $Z_{f}$, so

$$
\bar{S}_{\max }^{(b)}\left(Z_{f}, \mathrm{Oh}\right)=\max _{\bar{\omega}} \max _{1 \leq Z_{i}<Z_{f}} \bar{S}\left(Z_{i}, Z_{f}, \bar{\omega}, \mathrm{Oh}\right) .
$$




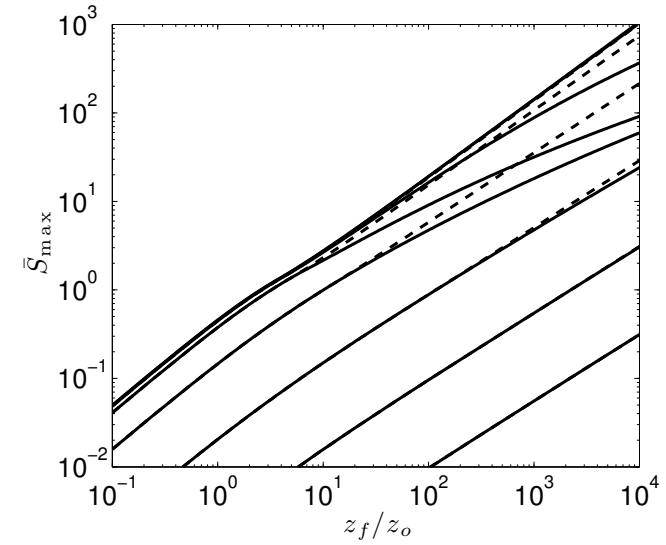

(a)

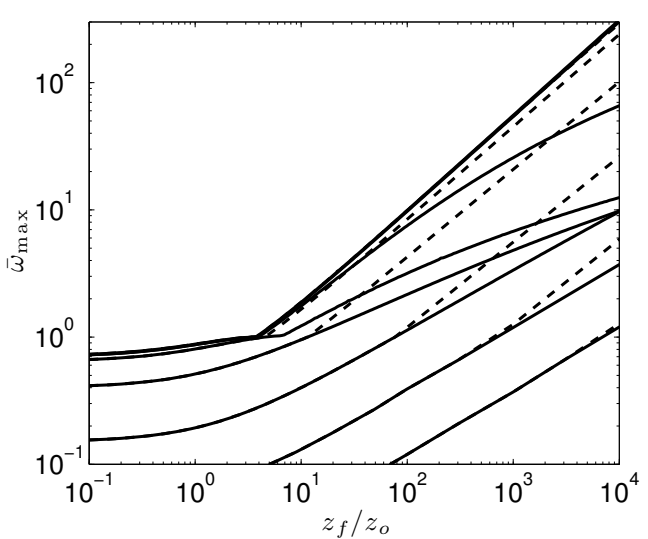

(b)

Figure 2: Maximum gain $\bar{S}_{\max }$ (a) and most dangerous frequency $\bar{\omega}_{\max }$ (b) of the perturbations excited from background noise (dashed lines) and at the nozzle (solid lines) as a function of the distance $z_{f} / z_{o}=Z_{f}-1$ to the nozzle. From bottom to top, Oh takes the values $1000,100,10,1,0.1,0.01,10^{-4}$.

For $z>1$, the integrand in the expression of $\bar{S}$ is always positive when $\bar{\omega}<1$. This means that as long as $\bar{\omega}_{\max }^{(a)} \leq 1$, the gain cannot be increased by changing $Z_{i}$, and we have $\bar{S}_{\max }^{(b)}=\bar{S}_{\max }^{(a)}$. When $\bar{\omega}_{\max }^{(a)}>1$, the perturbation starts to decrease before increasing further downstream. In that case, the gain can be increased by considering larger $Z_{i}$. More precisely, $Z_{i}$ has to be chosen such that the integrand starts to be positive which gives $Z_{i}=\bar{\omega}^{4 / 3}$. In this regime,

$$
\bar{S}_{\max }^{(b)}\left(Z_{f}, \mathrm{Oh}\right)=\max _{\bar{\omega}} \bar{S}\left(\bar{\omega}^{4 / 3}, Z_{f}, \bar{\omega}, \mathrm{Oh}\right) .
$$

Both $\bar{S}_{\max }^{(a)}$ and $\bar{S}_{\max }^{(b)}$ are obtained using standard Matlab subroutines.

\section{Quantitative results}

The results of the optimization procedure are shown in figure 2 for both nozzle excitation and background noise. Both the maximum gain and the most dangerous frequency are plotted versus the rescaled distance $z_{f} / z_{o}$ to the nozzle for Oh ranging from $10^{-4}$ to $10^{3}$. The same results are shown as level curves in the $\left(z_{f} / z_{o}, \mathrm{Oh}\right)$ plane in figure 3 . As expected, $\bar{S}_{\text {max }}$ grows as $z_{f} / z_{o}$ increases or Oh decreases (see figure $2($ a)). The most dangerous frequency follows the same trend (see figure 2(b)). As already mentioned above, nozzle excitation [case (a)] and background noise [case (b)] provide the same results when $\bar{\omega}_{\max } \leq 1$. The contour $\bar{\omega}_{\max }=1$ has been reported in figure $3(\mathrm{a})$ as a dotted line. On the left of this dotted line, the contours of maximum gain are then the same for both cases. When $\bar{\omega}_{\max }$ is larger than 1 , background noise gain becomes larger than nozzle excitation gain. The most dangerous frequency for background noise also becomes larger than for nozzle excitation. Note however that significant differences are only observed in an intermediate regime of $\mathrm{Oh}$ (typically $10^{-2}<\mathrm{Oh}<1$ ) for large values of $\bar{S}(\bar{S}>5)$ (see figure 3).

Figure 3 can be used to obtain the distance of the expected transition to jet breakup and droplet formation. Assume that a gain of order $G_{t} \approx e^{7}$, that is $S_{t}=7$ is enough for 


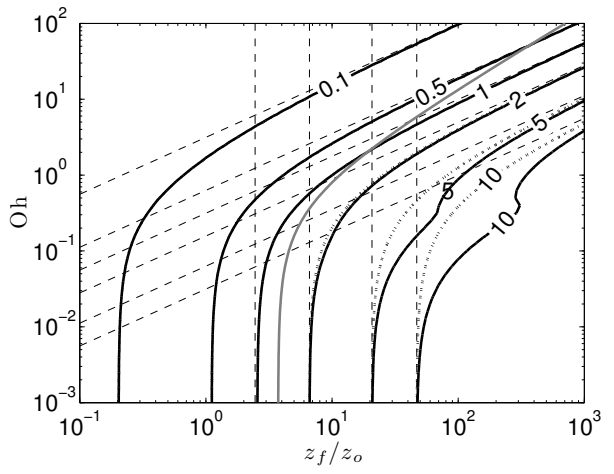

(a)

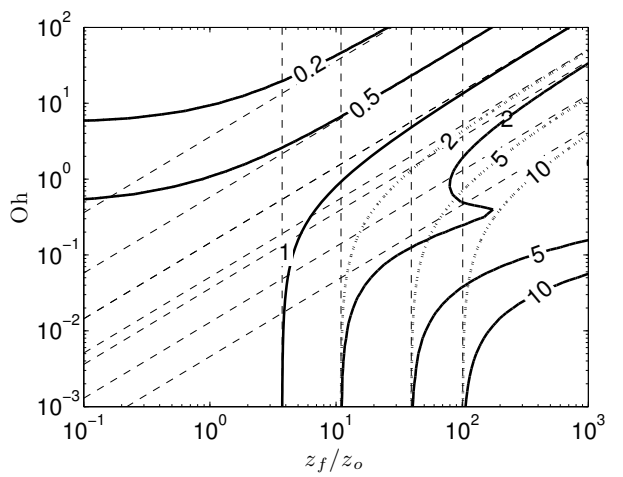

(b)

Figure 3: Level curves of the maximum gain $\bar{S}_{\max }$ (a) and of the most dangerous frequency $\bar{\omega}_{\max }(\mathrm{b})$ of the perturbations excited from background noise (dashed lines) and at the nozzle (solid lines) in the $\left(z_{f} / z_{o}, \mathrm{Oh}\right)$ plane. The dashed lines correspond to the asymptotic limits (3.11) and (3.5) for small and large Oh respectively. On the left of the $\omega_{\max }=1$ curve (indicated as a grey line in (a)), solid and dashed lines are superimposed.

the transition, a value commonly admitted in boundary layers instabilities (Schlichting 1987). From (2.26), we can deduce the value of $\bar{S}$ needed for transition

$$
\begin{aligned}
\bar{S}_{t} & =S_{t} \sqrt{2} Q / z_{o}=S_{t} 2 \sqrt{2} \text { Вo } / Q \\
& \approx 20 \text { Bo } / Q \\
& =20 \tau_{c} g / u_{0},
\end{aligned}
$$

and from figure 3(a) the position $z_{f} / z_{o}$ where such a value of $\bar{S}$ is reached in case (a) or (b).

If the fluid collapses in a single drop between two pinch-off, the distance between two droplets is given by the wavelength at breakup $\lambda_{\max }=2 \pi / A_{0}\left(z_{f}\right) / \bar{\omega}_{\max }$, deduced from (2.19), and the droplet diameter is

$$
d_{\max } \sim\left[6 \lambda_{\max } A_{0}\left(z_{f}\right)\right]^{1 / 3} \sim\left(\frac{12 \pi}{\bar{\omega}_{\max }}\right)^{1 / 3} .
$$

These two quantities are plotted in figure 4 for a few values of $\bar{S}_{t}$ as a function of Oh. What is particularly remarkable is that the drop diameter remains mostly constant in the full interval $10^{-3}<\mathrm{Oh}<10^{2}$ whatever the noise level for both cases [figure $4(\mathrm{~b})$ ]. Yet, in this interval of Oh, the breakup distance $z_{f}$ varies by a factor 1000 [figure 3(a)], while the wavelength varies by a factor 20 or more [figure 4(a)]. In the case of background noise, $z_{f}$ and $\lambda_{\max }$ increase with Oh. We observe the same evolution in the case of noise excitation for small $\bar{S}_{t}$. However, the curves of both cases depart from each other for large values of $\bar{S}_{t}$ (for instance $\bar{S}_{t}=10$ ) with a surprising local peak for case (a) close to $\mathrm{Oh} \approx 0.3$. As we shall see in section $\S 4$, this peak is associated with a larger damping of the perturbation outside the instability range for moderate $\mathrm{Oh}$.

In figures 3 and 4, we have also plotted the asymptotic behaviors of the different quantities obtained for large $\mathrm{Oh}$ and small Oh. The details of the derivation are provided in appendix A. We provide below the final result only. 


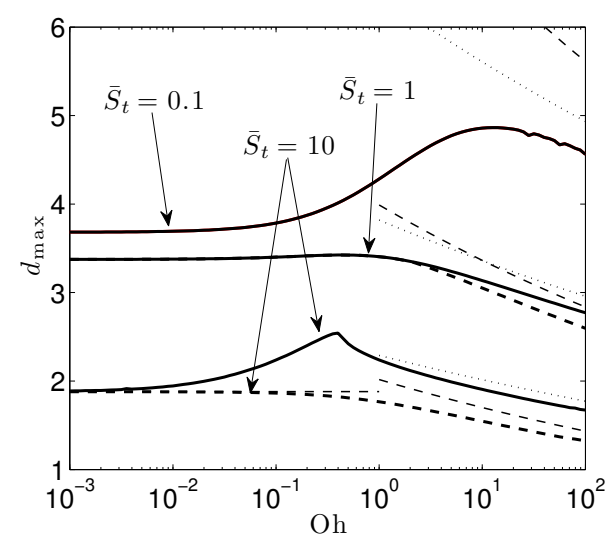

(a)

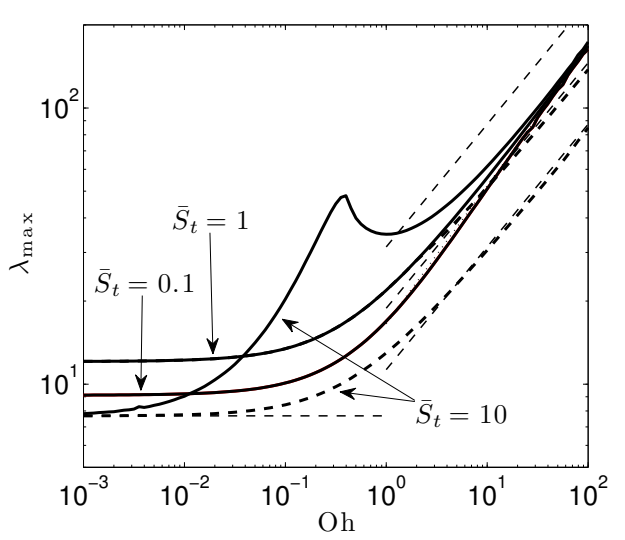

(b)

Figure 4: Wavelength at break-up (a) and resulting droplet diameter (b) versus Oh for background noise (dashed lines) and nozzle excitation (solid lines). The different curves correspond to the transition level $\bar{S}_{t}=0.1,1,10$. The thin dashed lines correspond to the asymptotic expressions for small and large $\mathrm{Oh}$.

\subsection{Large viscosity (large $\mathrm{Oh}$ )}

In the viscous regime $(\mathrm{Oh} \gg 1)$, the position $z_{f}$ of breakup strongly increases with $\mathrm{Oh}$, and converges to the asymptotic curve [deduced from (A 10) and (A 12)]

$$
z_{f} / z_{0} \sim\left(\frac{9}{2 \sqrt{2}}\right)^{4 / 3} \bar{S}_{t}^{4 / 3} \mathrm{Oh}^{4 / 3},
$$

for both cases (a) and (b) if $1 \ll z_{f} / z_{o} \ll \mathrm{Oh}^{4}$. The variation in terms of Bo and $Q$ can be obtained using (3.1) and (2.12).

This scaling law, which was also derived by Javadi et al. (2013), expresses that breakup occurs when the local capillary instability growth rate overcomes the stretching rate of the jet. Indeed and coming back to dimensional quantities, the velocity and local radius vary far from the nozzle as $U_{0} \sim \sqrt{2 g z}$ and $h \sim \sqrt{Q^{*}} /(2 g z)^{1 / 4}$, respectively where $Q^{*}=U_{0} h^{2}$ is the dimensional flow rate. The local stretching rate is then given by $\partial_{z} U_{0} \sim \sqrt{g /(2 z)}$ while the viscous capillary growth rate based on the current radius is of order $\gamma /(\eta h)=\gamma(2 g z)^{1 / 4} /\left(\eta \sqrt{Q^{*}}\right)$. The latter overcomes the former at a distance $z_{f}$ of order $(\eta / \gamma)^{4 / 3} g^{1 / 3}\left(Q^{*}\right)^{2 / 3}$. In terms of dimensionless parameters, this gives

$$
z_{f} / h_{0} \propto \mathrm{Oh}^{4 / 3} \mathrm{Bo}^{1 / 3} Q^{2 / 3}
$$

which is essentially the scaling deduced from (3.5) if one remembers that $\bar{S}_{t} \propto$ Bo $/ Q$ and $z_{0} \propto Q^{2} /$ Bo.

In that viscous regime, the most dangerous frequencies are not the same in cases (a) and (b). This implies that the wavelengths $\lambda_{\max }$ at the point of transition, and the droplet diameter $d_{\max }$ are also different. For case (a), we obtain from (A 9) and (3.5)

$$
\bar{\omega}_{\max }^{(a)} \sim \alpha_{a} \bar{S}_{t}^{2 / 3} \mathrm{Oh}^{1 / 6}, \text { with } \alpha_{a}=\frac{3^{3 / 4}}{2^{7 / 4}} \approx 0.678
$$


which gives

$$
\begin{aligned}
& \lambda_{\max }^{(a)} \sim \beta_{a} \mathrm{Oh}^{1 / 2}, \text { with } \beta_{a}=4 \pi 3^{1 / 4} \approx 16.54, \\
& d_{\max }^{(a)} \sim \gamma_{a} \bar{S}_{t}^{-2 / 9} \mathrm{Oh}^{-1 / 18}, \text { with } \gamma_{a}=\pi^{1 / 3} 3^{1 / 12} 2^{15 / 12} \approx 3.82 .
\end{aligned}
$$

For case (b), we obtain from (A 11) and (3.5)

$$
\bar{\omega}_{\max }^{(b)} \sim \alpha_{b} \bar{S}_{t}^{8 / 9} \mathrm{Oh}^{2 / 9}, \text { with } \alpha_{b}=\frac{3}{2^{7 / 3}} \approx 0.595
$$

which gives

$$
\begin{aligned}
& \lambda_{\max }^{(b)} \sim \beta_{b} \bar{S}_{t}^{-2 / 9} \mathrm{Oh}^{4 / 9}, \text { with } \beta_{b}=2^{31 / 12} \pi \approx 18.83, \\
& d_{\max }^{(b)} \sim \gamma_{b} \bar{S}_{t}^{-8 / 27} \mathrm{Oh}^{-2 / 27}, \text { with } \gamma_{b}=\pi^{1 / 3} 2^{13 / 9} \approx 3.99 .
\end{aligned}
$$

A naive local argument like the one leading to equation (3.6) would predict for $\lambda_{\max }$ the most unstable local wavelength at $z_{f}$. As it will be shown in section $\S 5$, this fails at making the correct predictions, precisely because it ignores the stretching history of the fluid particles, and of the corresponding unstable modes. Equation (3.6) is thus consistent with a local argument, but the local argument does not incorporate the whole truth.

\subsection{Low viscosity (small $\mathrm{Oh}$ )}

In the weakly viscous regime $(\mathrm{Oh} \ll 1)$, both noise and nozzle excitations are expected to give the same breakup distance $z_{f}$. This distance is well approximated by

$$
z_{f} / z_{0} \approx \eta_{0} \bar{S}_{t}^{8 / 7} \text { with } \eta_{0} \approx 3.45
$$

when $z_{f} / z_{o}>3.74$, that is $\bar{S}_{t}>1.32$.

Again, as in the previous viscous limit, this scaling law expresses that breakup occurs when the local capillary instability growth rate overcomes the stretching rate. The local jet stretching rate is still $\partial_{z} U_{0} \sim \sqrt{g /(2 z)}$ while the inviscid capillary growth rate based on the current radius is now of order $\sqrt{\gamma / \rho h^{3}}=\sqrt{\gamma / \rho}(2 g z)^{3 / 8} /\left(Q^{*}\right)^{3 / 4}$. The latter overcomes the former at a distance of order $\left(Q^{*}\right)^{6 / 7} g^{1 / 7}(\rho / \gamma)^{4 / 7}$. In terms of dimensionless parameters, it gives

$$
z_{f} / h_{0} \propto \mathrm{Bo}^{1 / 7} Q^{6 / 7},
$$

which is essentially the scaling in equation (3.11) with $\bar{S}_{t} \propto$ Bo $/ Q$ and $z_{0} \propto Q^{2} /$ Bo.

In this regime, the most dangerous frequency is also the same in both cases and given by

$$
\bar{\omega}_{\max }=\alpha_{0} \bar{S}_{t}^{6 / 7}, \text { with } \alpha_{0} \approx 0.79
$$

which gives

$$
\begin{aligned}
& \lambda_{\max } \sim \beta_{0} \bar{S}_{t}^{-2 / 7}, \quad \text { with } \beta_{0} \approx 14.82, \\
& d_{\max } \sim \gamma_{0} \bar{S}_{t}^{-2 / 7}, \quad \text { with } \gamma_{0} \approx 3.63 .
\end{aligned}
$$

Again and for the same reason, naive local scaling fails at representing these scaling laws adequately.

\section{Comparison with $3 \mathrm{D}$ predictions}

In this section, we focus on the regime of intermediate values of $\mathrm{Oh}$ for which the asymptotic expressions do not apply. We address the peculiar behavior of the optimal 


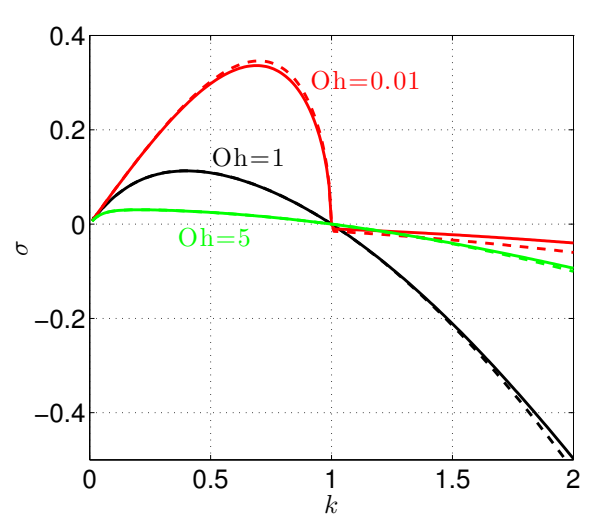

(a)

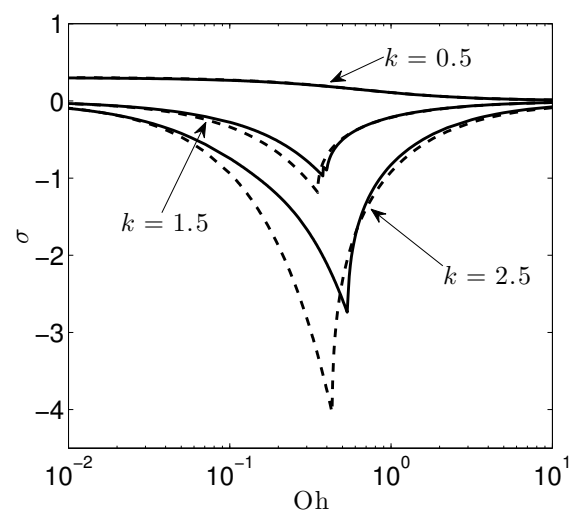

(b)

Figure 5: Comparison of $1 \mathrm{D}$ and 3D local dispersion relations. Solid line: 1D dispersion relation. Dashed line: 3D dispersion relation for axisymmetric modes. (a) Temporal growth rate versus the wavenumber $k$ for various Oh. (b) Temporal growth rate versus Oh for fixed wavelengths.

perturbation in the case of nozzle excitation in this regime. In figure $4(\mathrm{a}, \mathrm{b})$ ), we have seen that for $\bar{S}_{t}=10$ both $\lambda_{\max }$ and $d_{\max }$ exhibit a surprising kink around $\mathrm{Oh} \approx 0.3$. The same non-monotonic behavior has also been observed on the break-up distance $z_{f} / z_{0}$ as a function of $\mathrm{Oh}$ (see figure 3(a)). These surprising behaviors are associated with the particular properties of the perturbations outside the instability domain. Indeed, for large $\bar{S}_{t}$, the optimal perturbation is obtained for $\bar{\omega}_{\max }>1$. The local wavenumber of the perturbation which is $\bar{\omega}$ at the nozzle is then larger than 1 close to the nozzle, that is in the stable regime [see figure 5(a)]. The optimal perturbation excited from the nozzle is thus first spatially damped before becoming spatially amplified. This damping regime explains the smaller gain obtained by nozzle excitation compared to background noise. It turns out that the strength of this damping is not monotonic with respect to $\mathrm{Oh}$ and exhibits a peak for an intermediate value of Oh. Such a peak is illustrated in figure 5 where we have plotted the (local) temporal growth rate of the perturbation versus Oh for a few values of the (local) wavenumber. We do observe that for the values of $k$ satisfying $k \geq 1$, that is outside the instability band, the local growth rate exhibits a negative minimum for Oh between 0.1 and 1 .

The presence of this damping regime naturally questions the validity of our 1D model. The 1D model is indeed known to correctly describe the instability characteristics of 3D axisymmetric modes (Eggers \& Villermaux 2008). But, no such results exist in stable regimes. In fact, the $1 \mathrm{D}$ dispersion relation departs from the $3 \mathrm{D}$ dispersion relation of axisymmetric modes when $k>1$. This departure is visible in figure 5 where we have also plotted the local growth rate obtained from the $3 \mathrm{D}$ dispersion relation given in Chandrasekhar (1961), p. 541. Significant differences are observed but the 3D growth rates exhibit a similar qualitative behavior as a function of $\mathrm{Oh}$. In particular, there is still a damping rate extremum in the interval $0.1<\mathrm{Oh}<1$. We can therefore expect a similar qualitative behavior of the perturbation outside the instability range with the 3D model.

In figure 6, we compare the optimization results for the nozzle excitation obtained with the $1 \mathrm{D}$ model with those obtained using the $3 \mathrm{D}$ dispersion relation of Chandrasekhar. 


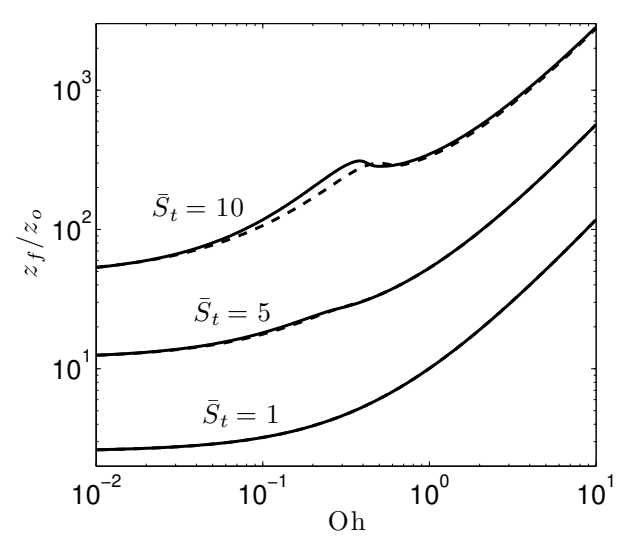

(a)

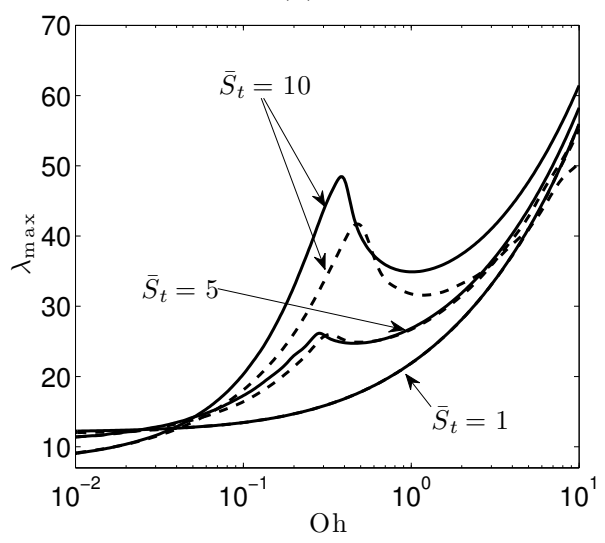

(c)

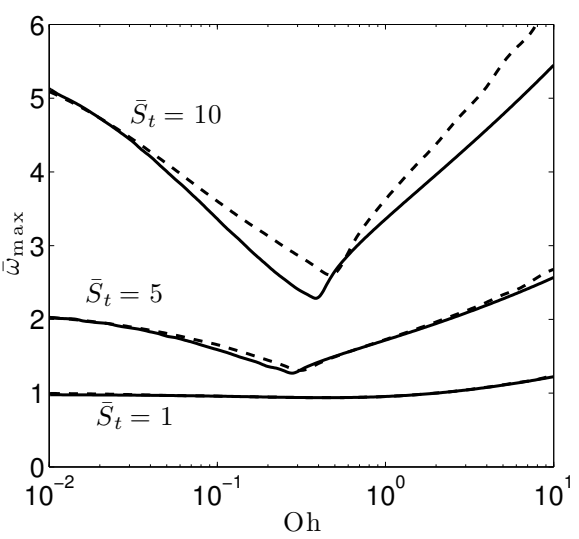

(b)

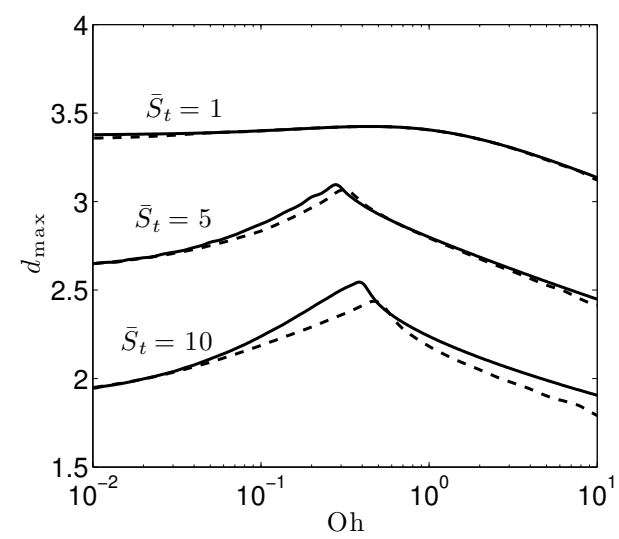

(d)

Figure 6: Characteristics of the response to nozzle excitation versus Oh for various values of $\bar{S}_{t}$ and two different stability models (Solid line: 1D; Dashed line: 3D axisymmetric). (a) Break-up distance. (b) Most dangerous frequency (c) Wavelength at break-up. (d) Drop diameter.

This is done by replacing the function $\bar{S}$ in $(2.27)$ by

$$
\bar{S}^{(3 D)}\left(Z_{i}, Z_{f}, \bar{\omega}, \mathrm{Oh}\right)=\sqrt{2} \mathrm{Oh} \int_{Z_{i}}^{Z_{f}}\left(y^{2}(x, J)-x^{2}\right) d z,
$$

where

$$
x=x(z, \bar{\omega})=\frac{\bar{\omega}}{z^{3 / 4}}, \quad J=J(z, \mathrm{Oh})=\frac{1}{\mathrm{Oh}^{2} z^{1 / 4}},
$$

and $y=y(x, J)$ is given by

$$
2 x^{2}\left(x^{2}+y^{2}\right) \frac{I_{1}^{\prime}(x)}{I_{0}(x)}\left[1-\frac{2 x y}{x^{2}+y^{2}} \frac{I_{1}(x) I_{1}^{\prime}(y)}{I_{1}(y) I_{1}^{\prime}(x)}\right]-\left(x^{4}-y^{4}\right)=J \frac{x I_{1}(x)}{I_{0}(x)}\left(1-x^{2}\right) .
$$

As expected, differences can be observed between 1D and 3D results for the largest value of $\bar{S}_{t}\left(\bar{S}_{t}=10\right)$. However, the trends remain the same. Close to $\mathrm{Oh} \approx 0.3$, the breakup distance exhibits a plateau, the frequency a minimum, the wavelength and the drop diameter a peak. These peaks have a smaller amplitude for the $3 \mathrm{D}$ dispersion relation 
and are slightly shifted to higher values of Oh. For $\bar{S}_{t}=1$, no difference between both models are observed. This can be understood by the fact that the perturbation does not exhibit a period of damping for such a small value of $\bar{S}_{t}$. The 1D model therefore perfectly describes the gain of 3D perturbations, which turns out to be the same as for background noise for $\mathrm{Oh}<2$ (see figure $3(\mathrm{a})$ ).

\section{Comparison with local predictions}

In this section, our goal is to compare the results of the optimization procedure with predictions obtained from the local dispersion relation. We have seen in section 2 that the gain can be related to the local temporal growth rate of the perturbation along the jet [see expression (2.23)]. Both the local capillary time scale $\tau_{c_{l}}$ and the Ohnesorge number $\mathrm{Oh}_{l}$ vary with $Z$ [see expressions $\left.(2.25 \mathrm{~b}, \mathrm{c})\right]$. At a location $Z$, the maximum temporal growth rate (normalized by the capillarity time at the nozzle) is given by

$$
\sigma_{l}^{\max }(Z)=\frac{Z^{3 / 8}}{2 \sqrt{2}+6 \mathrm{Oh} Z^{1 / 8}},
$$

and is reached for the wavelength (normalized by $h_{0}$ ) (see Eggers \& Villermaux 2008)

$$
\lambda_{l}(Z)=\frac{2 \pi}{Z^{1 / 4}} \sqrt{2+3 \sqrt{2} \mathrm{Oh} Z^{1 / 8}}
$$

If we form a drop from this perturbation wavelength at this location, we would then obtain a drop diameter (normalized by $h_{0}$ )

$$
d_{l}(Z)=\frac{(12 \pi)^{1 / 3}}{Z^{1 / 4}}\left(2+3 \sqrt{2} \mathrm{Oh} Z^{1 / 8}\right)^{1 / 6} .
$$

As the local growth rate increases downstream, a simple upperbound of the gain is then obtained by taking the exponential of the product of the maximum growth rate by the time $T_{i}$ needed to reach the chosen location. The time $T_{i}$ is the free fall time given by

$$
T_{i}=\frac{Q}{\mathrm{Bo}}(\sqrt{Z}-1)
$$

In figure 7 , we have plotted the product $\sigma_{l}^{\max } T_{i}$ at the location predicted for the transition assuming that a gain $e^{7}$ is needed for such a transition. In figure $7(\mathrm{a})$, this quantity is plotted as a function of the transition location $z_{f} / z_{o}$. As expected, we obtain the chosen value for the transition (i. e. 7) for small $z_{f} / z_{o}$. For large $z_{f} / z_{o}$, the product $\sigma_{l}^{\max } T_{i}$ also goes to a constant for background noise whatever the Ohnesorge number. However, it has a contrasted behavior for nozzle excitation, with an important increase with $z_{f} / z_{o}$ for the value $\mathrm{Oh}=0.3$.

In figure $7(\mathrm{~b}), \sigma_{l}^{\max } T_{i}$ is plotted as a function of $\mathrm{Oh}$, for different values of $\bar{S}_{t}$, that is for different values of the ratio $\mathrm{Bo} / Q$ in view of (3.1). For large and small $\mathrm{Oh}$ we recover the estimates deduced using (3.5) and (3.11):

$$
\begin{aligned}
\sigma_{l}^{\max } T_{i} \sim 10.5 & \text { as } \quad \mathrm{Oh} \rightarrow \infty, \\
\sigma_{l}^{\max } T_{i} \sim 20.68-11.14 \bar{S}_{t}^{-4 / 7} & \text { as } \quad \mathrm{Oh} \rightarrow 0 .
\end{aligned}
$$

For background noise, $\sigma_{l}^{\max } T_{i}$ varies smoothly between these two extreme values. A completely different evolution is observed for nozzle excitation: a local peak forms between $0.1<\mathrm{Oh}<1$ with an amplitude increasing with $\bar{S}_{t}$. This phenomenon is related to the damping of the optimal perturbation discussed in the previous section. We have indeed 


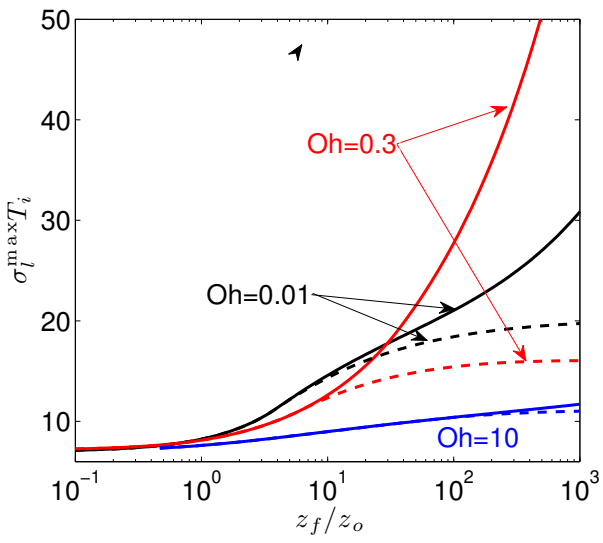

(a)

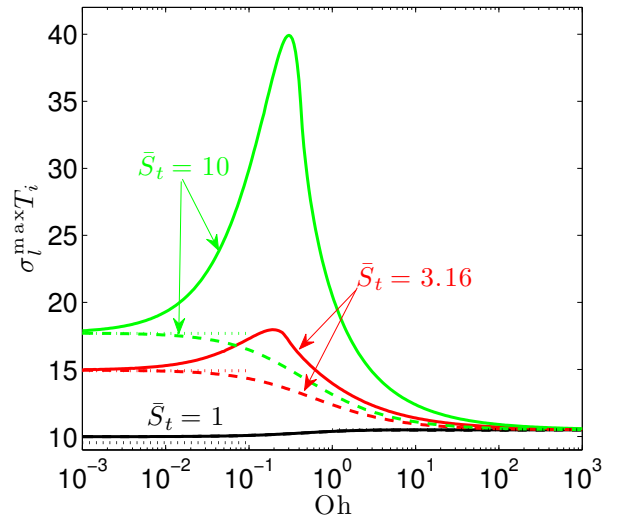

(b)

Figure 7: Maximum local temporal growth rate $\sigma_{l}^{\max }$ normalized by the free fall time $T_{i}$ at the breakup location assuming breakup for a gain $e^{7}$. Solid line: nozzle excitation; dashed line: background noise. (a) Variation with respect to the breakup location $z_{f} / z_{o}$ for different Oh. (b) Variation with respect to Oh for different values of $\bar{S}_{t}$. The dotted lines in (b) are the asymptotic predictions (5.5a,b).

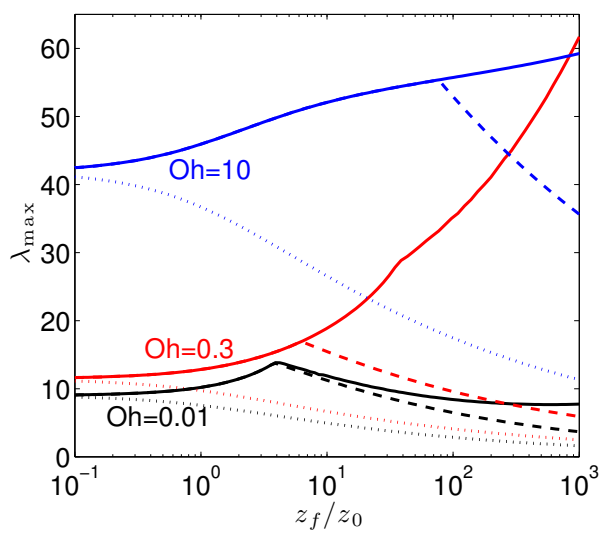

(a)

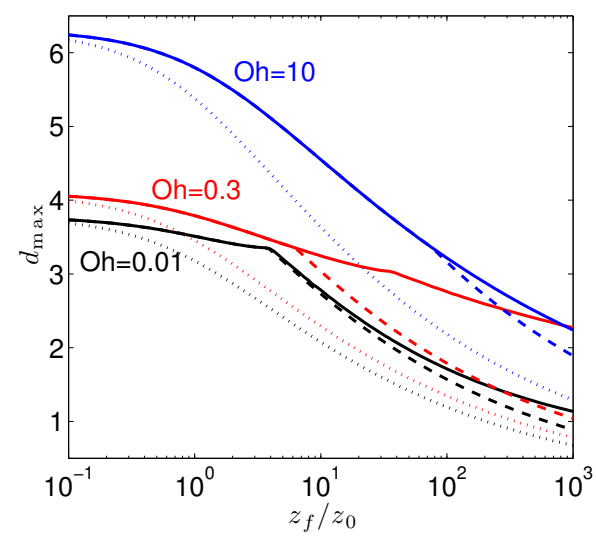

(b)

Figure 8: Wavelength at break-up (a) and drop diameter (b) versus the break-up location $z_{f} / z_{0}$ for various values of Oh. Solid line: nozzle excitation; dashed line: background noise.

seen that for nozzle excitation, large gain (that is large $\bar{S}_{t}$ ) are obtained for perturbations exhibiting a damping period prior to their growth. Thus, the growth has to compensate a loss of amplitude. The damping being the strongest for intermediate $\mathrm{Oh}$, the transition is pushed the farthest for these values, explaining the largest growth of the $\mathrm{Oh}=0.3$ curve in figure $7(\mathrm{a})$ and the peaks of figure $7(\mathrm{~b})$.

We have seen that the optimal procedure provides a wavelength and a droplet size as a function of $Z$ and $\mathrm{Oh}$ only. These quantities are compared to the local estimates (5.2) and (5.3) in figure 8. Both nozzle excitation (solid lines) and background noise (dashed lines) are considered for $\mathrm{Oh}=0.01,0.3$ and 10 . We observe that the local predictions (dotted lines) always underestimate the wavelength and the drop diameter. For the wavelength, the ratio with the local estimate typically increases with $z_{f} / z_{0}$ and Oh. The gap is the 
strongest for the nozzle excitation case, especially for intermediate $\mathrm{Oh}$ (see curve for $\mathrm{Oh}=0.3$ ) for which the local estimate is found to underestimate the wavelength by a factor as high as 25 for $z_{f} / z_{0}=10^{3}$.

Contrarily to the wavelength, the drop diameter follows the same trend as the local prediction as a function of $z_{f} / z_{0}$. For both noise excitation and background noise, the diameter decreases with the break-up location.

For large or small Oh, the behaviors of the wavelength and drop diameter obtained by the optimization procedure and local consideration can be directly compared using the results obtained in Appendix A. For large Oh, the local prediction reads

$$
\begin{array}{r}
\lambda_{l} / h_{0} \sim \beta_{l} \mathrm{Oh}^{1 / 2} Z_{f}^{-3 / 16}, \text { with } \beta_{l}=2 \pi 2^{1 / 4} \sqrt{3} \approx 12.94, \\
d_{l} / h_{0} \sim \gamma_{l} \mathrm{Oh}^{1 / 6} Z_{f}^{-11 / 49}, \text { with } \gamma_{l}=(2 \pi)^{1 / 3}(3 \sqrt{2})^{1 / 6} \approx 2.35
\end{array}
$$

while the optimization procedure gives

$$
\begin{aligned}
& \lambda_{\max }^{(a)} / h_{0} \sim \beta^{(a)} \mathrm{Oh}^{1 / 2}, \text { with } \beta^{(a)} \approx 16.54, \\
& \lambda_{\max }^{(b)} / h_{0} \sim \beta^{(b)} \mathrm{Oh}^{2 / 3} Z_{f}^{-1 / 6} \text {, with } \beta^{(b)} \approx 22.83 \text {, } \\
& d_{\max }^{(a)} / h_{0} \sim \gamma^{(a)} \mathrm{Oh}^{1 / 6} Z_{f}^{-1 / 6} \text {, with } \gamma^{(a)} \approx 4.63 \text {, } \\
& d_{\max }^{(b)} / h_{0} \sim \gamma^{(b)} \mathrm{Oh}^{2 / 9} Z_{f}^{-2 / 9}, \text { with } \gamma^{(b)} \approx 5.16
\end{aligned}
$$

For small Oh, the local estimates are

$$
\begin{array}{r}
\lambda_{l}^{n v} / h_{0} \sim \beta_{l}^{n v} Z_{f}^{-1 / 4}, \text { with } \beta_{l}^{n v} \approx 2 \pi \sqrt{2} \approx 8.88, \\
d_{l}^{n v} / h_{0} \sim \gamma_{l}^{n v} Z_{f}^{-1 / 4}, \quad \text { with } \gamma_{l}^{n v}=(12 \pi)^{1 / 3}(2)^{1 / 6} \approx 3.76,
\end{array}
$$

while the optimization procedure gives for $Z_{f}>4.74$ (see appendix A)

$$
\begin{gathered}
\lambda_{\max } / h_{0} \sim \beta^{n v} Z_{f}^{-1 / 4}, \text { with } \beta^{n v} \approx 20.20, \\
d_{\max } / h_{0} \sim \gamma^{n v} Z_{f}^{-1 / 4}, \text { with } \gamma^{n v} \approx 4.94 .
\end{gathered}
$$

\section{Applications}

We now apply the results to a realistic configuration obtained from an nozzle of radius $h_{0}=1 \mathrm{~mm}$ in a gravity field with $g=9.81 \mathrm{~m} / \mathrm{s}^{2}$. We consider three fluids: water (at $20^{\circ}$ ) for which $\gamma \approx 7210^{-3} \mathrm{~N} / \mathrm{m}, \nu \approx 10^{-6} \mathrm{~m}^{2} / \mathrm{s}$; and two silicon oils of surface tension $\gamma \approx 2110^{-3} \mathrm{~N} / \mathrm{m}$ and of viscosity $\nu \approx 510^{-5} \mathrm{~m}^{2} / \mathrm{s}$ and $\nu \approx 310^{-4} \mathrm{~m}^{2} / \mathrm{s}$ respectively. For these three fluids, we take $\rho \approx 10^{3} \mathrm{~kg} / \mathrm{m}^{3}$ as a fair order of magnitude.

For water, we obtain $\mathrm{Oh}=3.710^{-3}$, Bo $=0.13$ and a parameter $Q=3.72 u_{0}$ with the velocity $u_{0}$ at the nozzle expressed in $\mathrm{m} / \mathrm{s}$. For the silicon oils, we get Bo $=0.46$ and $Q=6.9 u_{0}$ and two values of $\mathrm{Oh}: \mathrm{Oh}=0.46$ and $\mathrm{Oh}=2$. The conditions of validity $(2.9 \mathrm{a}-\mathrm{c})$ of the inertial solution then require $u_{0}$ to be (much) larger than $u_{c}=0.26 \mathrm{~m} / \mathrm{s}$ for the water, and $u_{c}=0.15 \mathrm{~m} / \mathrm{s}$ for the silicon oils.

In figure 9 , we have plotted the theoretical predictions for the breakup location, the frequency, the wavelength and the drop diameter as the fluid velocity at the nozzle is varied from $u_{c}$ to $10 u_{c}$, that is for $Q$ varying from 1 to 10 . We have chosen $S_{t}=7$ for the background noise transition, and $S_{t}=4$ for the transition by the nozzle excitation. A smaller value of $S_{t}$ has been chosen for the nozzle excitation to describe controlled conditions of forcing. Figure 9 (a) shows that for the three fluids the transition by the nozzle excitation can be reached before the background noise transition. The values 


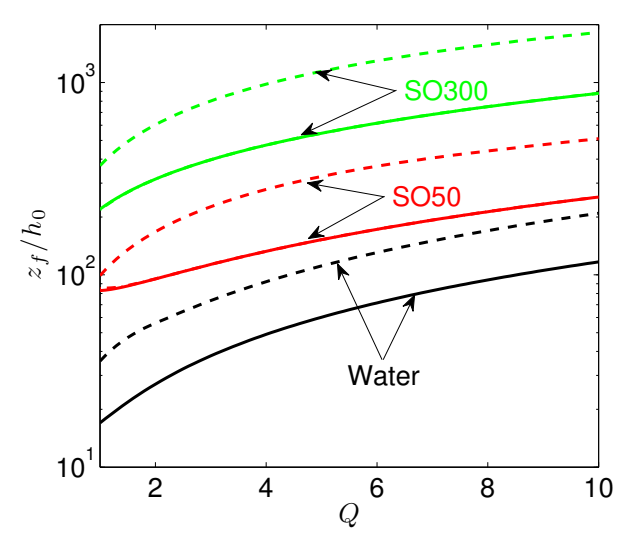

(a)

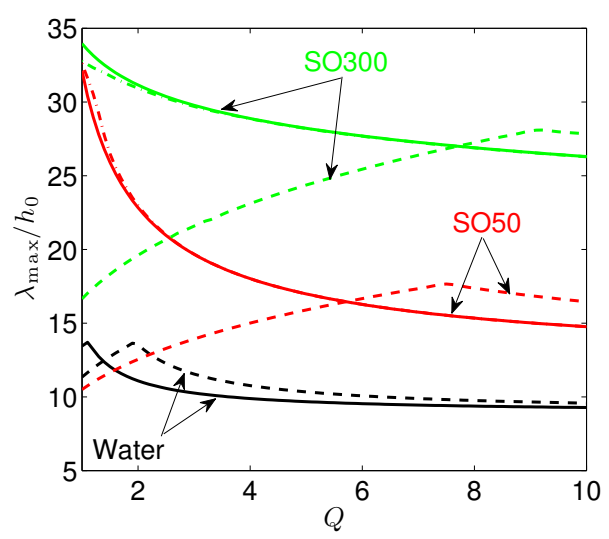

(c)

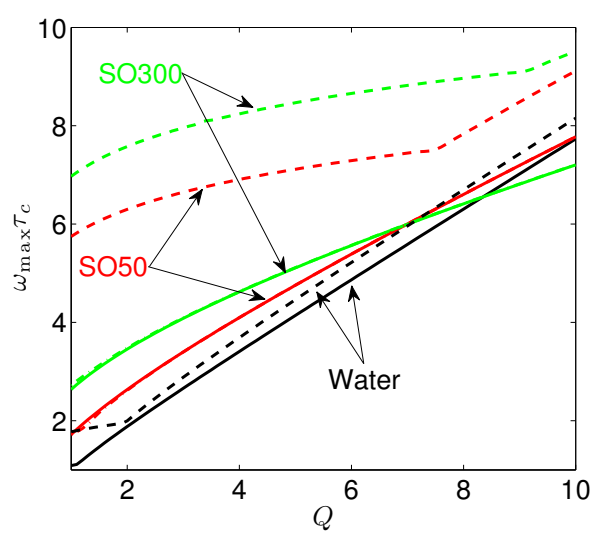

(b)

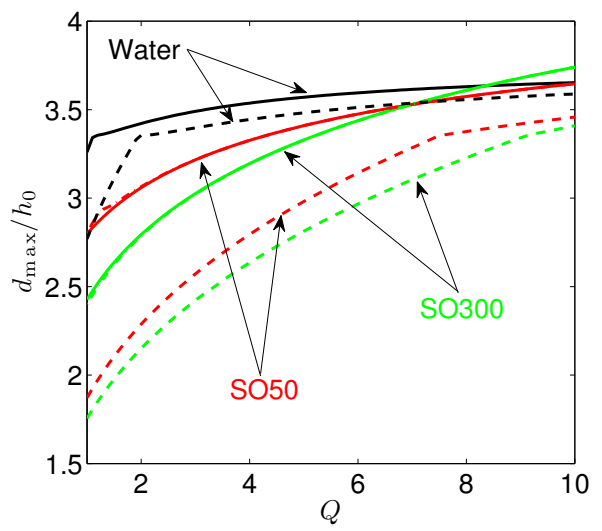

(d)

Figure 9: Characteristics at break-up by nozzle excitation or background noise for a jet of radius $h_{0}=1 \mathrm{~mm}$ assuming that break-up occurs when the perturbation gain has reached $e^{S_{t}}$. Solid lines: Nozzle excitation with $S_{t}=4$. Dot-dash lines: Nozzle excitation with $S_{t}=4$ using the 3D dispersion relation. Dashed lines: Background noise with $S_{t}=7$. Black lines: Water; Red lines: Silicon oil of $\nu=510^{-5} \mathrm{~m}^{2} / \mathrm{s}$ (SO50); Green lines: Silicon oil of $\nu=310^{-4} \mathrm{~m}^{2} / \mathrm{s}$ (SO300); (a): Break-up location; (b): Most dangerous frequency; (c): Wavelength at break up; (d): Drop diameter.

obtained for the breaking length are comparable to the experimental values reported in Javadi et al. (2013). They measured a normalized breaking length of order 100-150 for the silicon oil of $\nu \approx 510^{-5} \mathrm{~m}^{2} / \mathrm{s}$ from a nozzle of same diameter for flow rates ranging from $Q=0.5$ to $Q=1.3$.

Figure 9(b) provides the most dangerous frequency of the excitation. For the three cases, the frequency for the nozzle excitation is relatively closed to the neutral frequency $Q$ of the jet at the nozzle. For both silicon oils, this frequency is however much smaller than the frequency obtained by the background noise transition, especially for small $Q$.

The break-up wavelength shown in figure 9 (c) exhibits a different behavior with respect to the flow rate $Q$ for the nozzle excitation and the background noise. It decreases monotonically with $Q$ for the noise excitation while it increases for the background noise up to an extremum before starting decreasing. For the three fluids, noise excitation provides a 
larger wavelength than background noise for small $Q$, but the opposite is observed above a critical value of $Q$ which increases with Oh. Note that for small $Q$, the wavelengths obtained for noise excitation are comparable for both silicon oils. Both curves would even cross if a larger value of $S_{t}$ was considered. This property is related to the non-monotonic behavior of the breakup wavelength already discussed above [see figure 6(c)].

Contrarily to the wavelength, the droplet size [figure $9(\mathrm{~d})$ )] is not changing much with $Q$ and is comparable for the three fluids. Nozzle excitation provides larger droplets but this effect is significant for the smallest values of $Q$ only.

Finally note that the differences between the $1 \mathrm{D}$ and 3D predictions for the nozzle excitation are barely visible. A very small departure of the wavelength curves can be seen for the silicon oils only. This confirms both the usefulness and the validity of the $1 \mathrm{D}$ model.

\section{Conclusion and final remarks}

At the end of this detailed study, we are now in position to answer the questions raised in the Introduction: The breakup distance from the orifice of a jet falling by its own weight can indeed be understood by comparing two timescales. The relevant timescales are the capillary destabilization time (viscous, or not) based on the local jet radius, and the inverse of the local jet stretching rate. Breakup occurs, in both viscous and inviscid régimes as discussed in section 3, when the latter overcomes the former, a fact already known (Villermaux 2012; Javadi et al. 2013). However, we have also learned that this aspect is only a tiny piece of the problem as a whole. This simple local rule, if naively extended to estimate the wavelength of the perturbation breaking the jet would predict that the wavelength is proportional to the local jet radius in the inviscid case for instance. This prediction was found to always underestimate the wavelength at breakup. The most dangerous wavelength and the drop diameter account for the stretching history of the fluid particles as they travel along the jet; this is the reason why their values are different depending on whether the perturbations are introduced at the jet nozzle only, or through a background noise affecting the jet all along its extension. An optimal theory computing the gain of every mode as the jet deforms and accelerates, was thus necessary to answer the -seemingly simple- question of its breakup. It has, in addition, revealed the existence of an unexpected non-monotonic dependency of the most dangerous wavelength $\lambda_{\max }$ with respect to $\mathrm{Oh}$.

We have also provided quantitative results assuming that a spatial gain of $e^{7}$ of the linear perturbations was sufficient for breakup. This value of the critical gain is an ad hoc criterion that assumes a particular level of noise and which neglects the possible influence of the nonlinear effects. It would be interested to test this criterion with experimental data.

Our analysis has focused on capillary jet whose base state is in an inertial regime. Close to the nozzle, especially if the flow rate is small, a viscous dominated regime is expected (Senchenko \& Bohr 2005). We have not considered such a regime here. But a similar WKBJ analysis could a priori be performed with a base flow obtained by resolving the more general equations (2.2) if the jet variation scale remains large compared to the perturbation wavelength. However, far from the nozzle, the jet always becomes inertial. The growth of the perturbation is therefore expected to be the same as described above. For this reason, the optimal perturbation obtained from background noise could be the same. Indeed, we have seen that in order to reach a large gain $\left(\bar{S}_{t}>5\right.$ or so), the optimal perturbation should be introduced far from the nozzle. If the jet is in the inertial regime 
at this location, the same gain is then obtained. This point was already noticed in Javadi et al. (2013).

For nozzle excitation, the entire evolution of the jet contributes the optimal perturbation. We have seen that large gains $\left(\bar{S}_{t}>5\right)$ are obtained by perturbations which exhibit a spatial damping before starting to grow. We have also seen that this damping regime is only qualitatively described by the $1 \mathrm{D}$ model. We do not expect a better description if the jet is dominated by viscous effects. Moreover, it is known that in this regime nonparallel effects are also important close to the nozzle (Rubio-Rubio et al. 2013) which invalidates the WKBJ approach. For this regime, it would be interesting to perform an optimal stability analysis using more advanced tools (Schmid 2007) to take into account non-parallel effects and non-modal growth.

Note finally that we have computed the perturbation gain by considering the exponential terms of the WKBJ approximation only. A better estimate could readily be obtained by considering the complete expression of the WKBJ approximation. This expression which has been provided in appendix B involves an amplitude factor which contains all the other contributions affecting the growth of the perturbation. Different expressions are obtained for $A$ and $u$ which in particular implies that different gains are obtained for the velocity and the jet radius. It is important to mention that the other contributions are not limited to a simple correcting factor associated with the local stretching (Tomotika 1936; Eggers \& Villermaux 2008). Other contributions associated with the $z$-dependence of the local wavenumber and local jet profile are equally important, leading to expressions which are not simple even in the large or small Oh limit.

\section{Acknowledgments}

We acknowledge support from the French Agence Nationale de la Recherche under the ANR FISICS project ANR-15-CE30-0015-03.

\section{Appendix A. Asymptotic regimes}

In this appendix, we provide asymptotic expressions for $\bar{S}_{\max }$ and $\bar{\omega}_{\max }$ in the viscous and inviscid regimes, that is for $\mathrm{Oh} \rightarrow \infty$ and $\mathrm{Oh} \rightarrow 0$ respectively.

\section{A.1. Maximum gain in the viscous regime $(\mathrm{Oh} \rightarrow \infty)$}

When $\mathrm{Oh} \rightarrow \infty$, the expression of the integrand in (2.27) can be simplified, and in the whole domain of integration, we can use the approximation

$$
\sqrt{1-\bar{\omega}^{2} z^{-3 / 2}+\frac{9 \mathrm{Oh}^{2} \bar{\omega}^{2}}{2} z^{-5 / 4}} \sim \sqrt{1+\frac{9 \mathrm{Oh}^{2} \bar{\omega}^{2}}{2} z^{-5 / 4}}-\frac{\bar{\omega}^{2} z^{-3 / 2}}{2 \sqrt{1+\frac{9 \mathrm{Oh}^{2} \bar{\omega}^{2}}{2} z^{-5 / 4}}}
$$

such that $(2.27)$ can be written as

$$
\bar{S} \sim \frac{\bar{S}_{1}\left(Z_{i}, Z_{f}, X_{\omega}\right)}{\mathrm{Oh}}-\frac{\bar{S}_{2}\left(Z_{i}, Z_{f}, X_{\omega}\right)}{\mathrm{Oh}^{3}},
$$


with

$$
\begin{aligned}
\bar{S}_{1}\left(Z_{i}, Z_{f}, X_{\omega}\right) & =X_{\omega}^{-3 / 4} \int_{Z_{i} X_{\omega}}^{Z_{f} X_{\omega}} X^{-7 / 8}\left(\sqrt{1+\frac{9}{2 X^{5 / 4}}}-\frac{3}{\sqrt{2} X^{5 / 8}}\right) d X \\
\left.\bar{S}_{2}\left(Z_{i}, Z_{f}, X_{\omega}\right)\right) & =X_{\omega}^{-1 / 2} \int_{Z_{i} X_{\omega}}^{Z_{f} X_{\omega}} \frac{X^{-19 / 8}}{2 \sqrt{1+\frac{9}{2 X^{5 / 4}}}} d X
\end{aligned}
$$

and

$$
X_{\omega}=(\mathrm{Oh} \bar{\omega})^{-8 / 5}
$$

When $Z_{f}$ is not too large, we are in a configuration where:

(1) $Z_{i} X_{\omega} \ll 1$ and $Z_{f} X_{\omega} \ll 1$. In that case, we can write

$$
\begin{aligned}
\bar{S}_{1} \sim \frac{2 \sqrt{2}}{9}\left(Z_{f}^{3 / 4}-Z_{i}^{3 / 4}\right) & -\frac{X_{\omega}^{5 / 4}}{108 \sqrt{2}}\left(Z_{f}^{2}-Z_{i}^{2}\right)+O\left(X_{\omega}^{5 / 2} Z_{f}^{13 / 4}\right) \\
\bar{S}_{2} & \sim \frac{2 \sqrt{2}}{9 X_{\omega}^{5 / 4}}\left(\frac{1}{Z_{i}^{3 / 4}}-\frac{1}{Z_{f}^{3 / 4}}\right)+O\left(Z_{f}^{1 / 2}\right)
\end{aligned}
$$

which gives

$$
\bar{S} \sim \frac{2 \sqrt{2}}{9 \mathrm{Oh}}\left(Z_{f}^{3 / 4}-1-\bar{\omega}^{2}+\bar{\omega}^{2} Z_{f}^{-3 / 4}\right)-\frac{Z_{f}^{2}-1}{108 \sqrt{2} \mathrm{Oh}^{3} \bar{\omega}^{2}}+O\left(\frac{Z_{f}^{1 / 2}}{\mathrm{Oh}^{3}}, \frac{Z_{f}^{13 / 4}}{\mathrm{Oh}^{5} \bar{\omega}^{4}}\right)
$$

in case (a) (nozzle excitation) and

$$
\bar{S} \sim \frac{2 \sqrt{2}}{9 \mathrm{Oh}}\left(Z_{f}^{3 / 4}-2 \bar{\omega}+\bar{\omega}^{2} Z_{f}^{-3 / 4}\right)-\frac{Z_{f}^{2}-\bar{\omega}^{8 / 3}}{108 \sqrt{2} \mathrm{Oh}^{3} \bar{\omega}^{2}}+O\left(\frac{Z_{f}^{1 / 2}}{\mathrm{Oh}^{3}}, \frac{Z_{f}^{13 / 4}}{\mathrm{Oh}^{5} \bar{\omega}^{4}}\right)
$$

in case (b) (background noise) with $Z_{i}=\bar{\omega}^{4 / 3}$.

In case (a), the maximum gain is obtained for

$$
\bar{\omega}_{\max }^{(a)} \sim\left(\frac{Z_{f}^{2}-1}{48 \mathrm{Oh}^{2}\left(1-Z_{f}^{-3 / 4}\right)}\right)^{1 / 4}
$$

that is

for large $Z_{f}$, and equals

$$
\bar{\omega}_{\max }^{(a)} \sim \frac{Z_{f}^{1 / 2}}{23^{1 / 4} \mathrm{Oh}^{1 / 2}}
$$

$$
\bar{S}_{\max }^{(a)} \sim \frac{2 \sqrt{2} Z_{f}^{3 / 4}}{9 \mathrm{Oh}}\left(1-Z_{f}^{-3 / 4}-\frac{Z_{f}^{1 / 4}}{2 \sqrt{3} \mathrm{Oh}}\right)+O\left(\frac{Z_{f}^{1 / 4}}{\mathrm{Oh}^{2}}, \frac{Z_{f}^{5 / 4}}{\mathrm{Oh}^{3}}\right) .
$$

In case (b), the maximum gain is obtained for

$$
\bar{\omega}_{\max }^{(b)} \sim \frac{Z_{f}^{2 / 3}}{48^{1 / 3} \mathrm{Oh}^{2 / 3}}
$$

and equals

$$
\bar{S}_{\max }^{(b)} \sim \frac{2 \sqrt{2} Z_{f}^{3 / 4}}{9 \mathrm{Oh}}\left(1-\frac{3^{2 / 3}}{2^{4 / 3} \mathrm{Oh}^{2 / 3} Z_{f}^{1 / 12}}\right)
$$




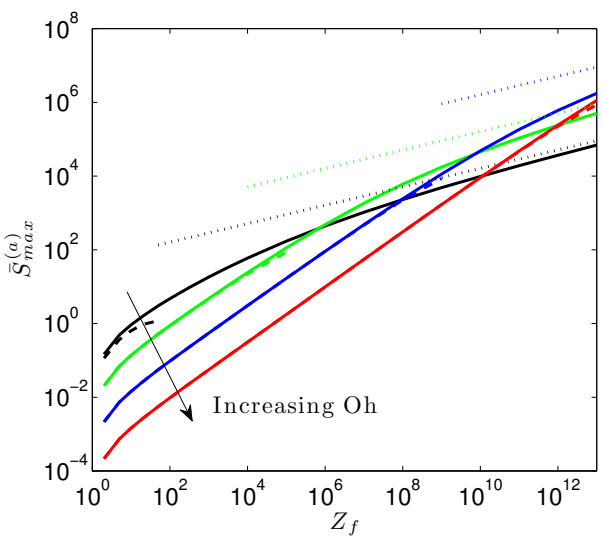

(a)

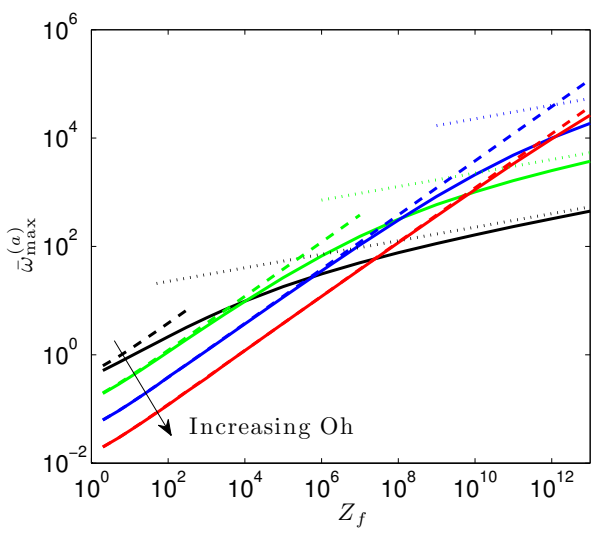

(b)

Figure 10: Maximum gain $\bar{S}_{\max }^{(a)}$ (a) and most dangerous frequency $\bar{\omega}_{\max }^{(a)}$ (b) of the perturbations excited at the nozzle as a function of the distance $Z_{f}$ to the nozzle. Solid lines: numerical results. Dashed and dotted lines: asymptotic results obtained for large Oh for $Z_{f} \ll \mathrm{Oh}^{4}$ [formulae (A 10) and (A 9)] and $Z_{f} \gg \mathrm{Oh}^{4}$ [formulae (A 17) and (A 16)] respectively. Oh takes the values $1,10,100,1000$.

The condition that $Z_{f} X_{\omega}^{\max } \ll 1$ does not give any restriction in case (b). However, it requires in case (a)

$$
Z_{f} \ll \mathrm{Oh}^{4}
$$

When $Z_{f} \gg \mathrm{Oh}^{4}$, another limit has to be considered for case (a): (2) $Z_{i} X_{\omega} \ll 1$ and $Z_{f} X_{\omega} \gg 1$. In this limit, we have

$$
\begin{gathered}
\bar{S}_{1} \sim \frac{8 Z_{f}^{8}}{X_{\omega}^{5 / 8}}-\frac{I_{o}}{X_{\omega}^{3 / 4}} \\
\bar{S}_{2} \sim \frac{2 \sqrt{2}}{9 X_{\omega}^{5 / 4} Z_{i}^{3 / 4}}
\end{gathered}
$$

It gives

$$
\bar{S} \sim 8 Z_{f}^{1 / 8} \bar{\omega}-I_{o} \bar{\omega}^{6 / 5} \mathrm{Oh}^{1 / 5}-\frac{2 \sqrt{2} \bar{\omega}^{2}}{9 \mathrm{Oh}}+O\left(\frac{Z_{f}^{1 / 2}}{\mathrm{Oh}^{3}}, \frac{Z_{f}^{13 / 4}}{\mathrm{Oh}^{5} \bar{\omega}^{4}}\right)
$$

which is maximum for

The maximum gain equals

$$
\bar{\omega}_{\max }^{(a)} \sim 9 \sqrt{2} \mathrm{Oh} Z_{f}^{1 / 8}
$$

$$
\bar{S}_{\max }^{(a)} \sim 36 \sqrt{2} Z_{f}^{1 / 4} \mathrm{Oh}
$$

This estimate applies only when $Z_{f} \gg \mathrm{Oh}^{4}$.

The asymptotic formulae are compared to numerical results in figures 10 for case (a) and in figure 11 for case (b). In both cases, we have plotted the maximum gain $\bar{S}_{\max }$ and the most dangerous frequency (the frequency that provides the maximum gain) versus $Z_{f}$ for $\mathrm{Oh}=1,10,100,1000$. It is interesting to see that in case (a) the maximum gain and the most dangerous frequency both collapse on a single curve when plotted as a function of the variable $Z_{f} / \mathrm{Oh}^{4}$ with an adequate normalization (see figure 12). 


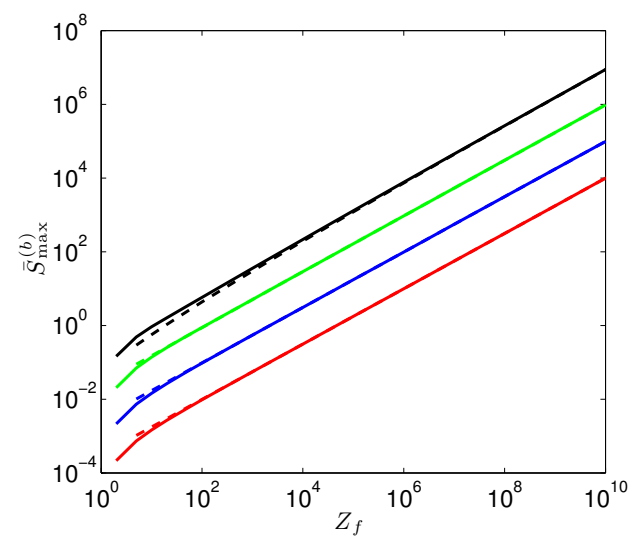

(a)

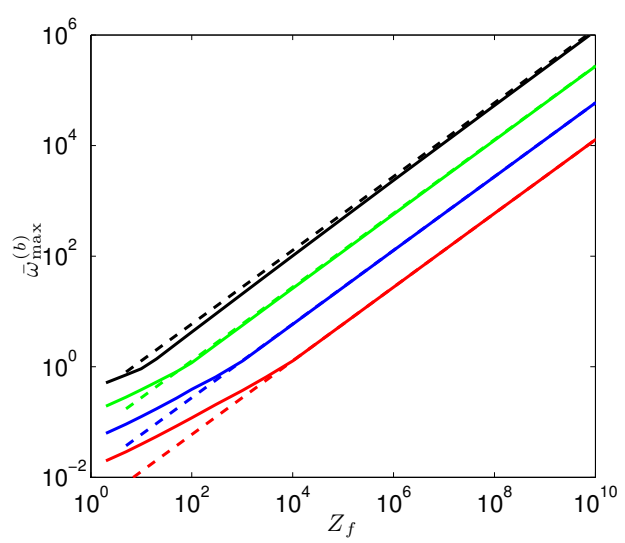

(b)

Figure 11: Maximum gain $\bar{S}_{\max }^{(b)}$ (a) and most dangerous frequency $\bar{\omega}_{\max }^{(b)}$ (b) of the perturbations excited from background noise as a function of the distance $Z_{f}$ to the nozzle. Solid lines: numerical results. Dashed lines: asymptotic results [formulae (A 12) and (A 11)]. From top to bottom, Oh takes the values 1, 10, 100, 1000.

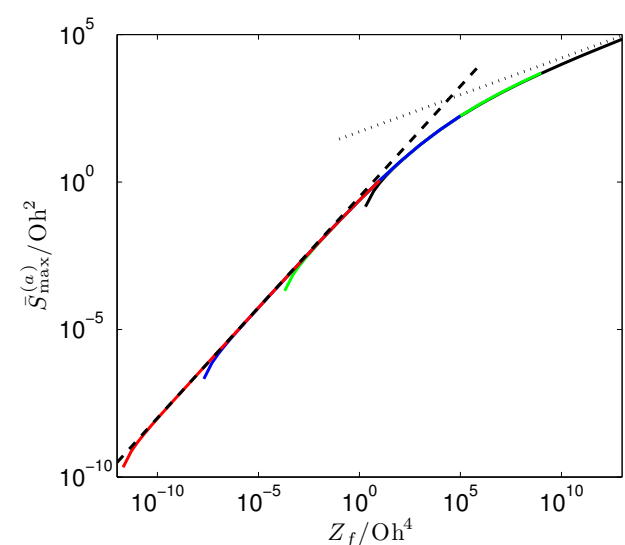

(a)

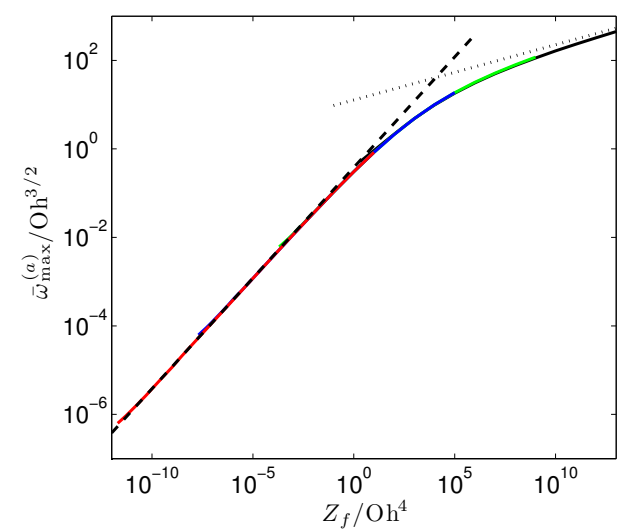

(b)

Figure 12: Same plots as fig. 10 but with rescaled variables versus $Z_{f} / \mathrm{Oh}^{4}$. In (a), the dotted line is (A 17) while the dashed line is the first term of (A 10). In (b), the dotted and dashed line are (A 16) and (A 9) respectively.

\section{A.2. Maximum gain in the inviscid regime $(\mathrm{Oh} \rightarrow 0)$}

When Oh is small, viscous effects come into play if we go sufficiently far away for the nozzle because the local Ohnesorge number increases algebraically with the distance to the nozzle.

Here, we shall assume that we remain inviscid in the whole domain of integration, that is

$$
\sqrt{1-\bar{\omega}^{2} z^{-3 / 2}+\frac{9 \mathrm{Oh}^{2} \bar{\omega}^{2}}{2} z^{-5 / 4}}-\frac{3}{\sqrt{2}} \bar{\omega} \mathrm{Oh} z^{-5 / 8} \sim \sqrt{1-\bar{\omega}^{2} z^{-3 / 2}}
$$




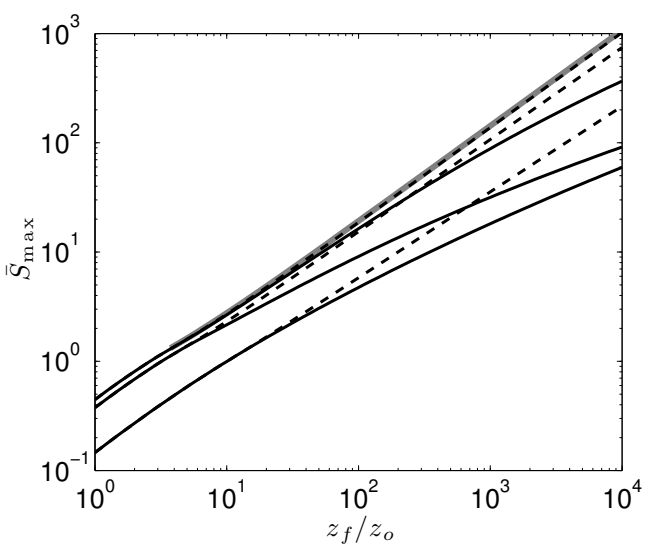

(a)

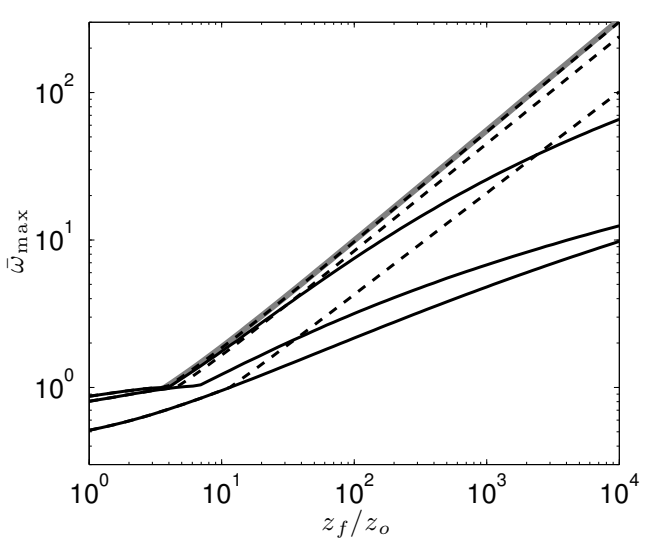

(b)

Figure 13: Maximum gain $\bar{S}_{\max }$ (a) and most dangerous frequency $\bar{\omega}_{\max }$ (b) of the perturbations excited from background noise (dashed lines) and at the nozzle (solid lines )as a function of the distance $z_{f} / z_{o}=Z_{f}-1$ to the nozzle. From bottom to top, Oh takes the values 0.1,0.01,0.001. The formulae (A 22) and (A 21) are indicated as a solid gray line in (a) and (b) respectively.

This is satisfied if $Z_{f} \ll \mathrm{Oh}^{-8}$. The gain can then be written as

$$
\bar{S} \sim Y_{\omega}^{-7 / 8} \int_{Z_{i} Y_{\omega}}^{Z_{f} Y_{\omega}} s^{-7 / 8} \sqrt{1-s^{-3 / 2}} d s
$$

with $Y_{\omega}=\bar{\omega}^{-4 / 3}$.

Because in the inviscid limit, perturbations are neutral when they do not grow, case (a) and case (b) provide the same gain.

For $1<Z_{f}<Z_{f}^{c} \approx 4.74$, the maximum gain is reached for $\bar{\omega}<1$, i.e. $Y_{\omega}>1$. The location $Z_{f}^{c}$ is given by the vanishing of $\partial_{Y_{\omega}} \bar{S}$ for $Y_{\omega}=1$ and $Z_{i}=1$ :

$$
-\frac{7}{8} \int_{1}^{Z_{f}^{c}} s^{-7 / 8} \sqrt{1-s^{-3 / 2}} d s+\left(Z_{f}^{c}\right)^{1 / 8} \sqrt{1-\left(Z_{f}^{c}\right)^{-3 / 2}}=0 .
$$

For $Z_{f}^{c}<Z_{f} \ll \mathrm{Oh}^{-8}$, the maximum gain is reached for

$$
\bar{\omega}_{\max } \sim\left(\frac{Z_{f}}{Z_{f}^{c}}\right)^{3 / 4} \approx 0.311 Z_{f}^{3 / 4}
$$

and equals

$$
\bar{S}_{\max }=\beta Z_{f}^{7 / 8}
$$

where

$$
\beta=\left(Z_{f}^{c}\right)^{-7 / 8} \int_{1}^{Z_{f}^{c}} s^{-7 / 8} \sqrt{1-s^{-3 / 2}} d s \approx 0.338 .
$$

This estimate is compared to numerical values in figure 13 . We do observe a convergence of the maximum gain and most dangerous frequency curves toward the inviscid limit as Oh decreases. Note however that the convergence is slower for nozzle excitation (case (a)). 


\section{Appendix B. WKBJ analysis}

In this section, we provide the full expression of the WKBJ approximation of each downward propagative wave. Each wave is searched into the form

$$
\left(u_{p}, A_{p}\right)=(v(Z), a(Z)) e^{i z_{o} \int^{Z} k(s) d s} e^{-i \omega t},
$$

where $k(Z), v(Z)$ and $a(Z)$ depend as the base flow on the slow spatial variable $Z=$ $z / z_{o}+1, z_{o}=Q^{2} /(2 \mathrm{Bo})$ being a large parameter.

If we plug expression (B 1) into (2.13), we get up to $O\left(1 / z_{o}^{2}\right)$ terms

$$
\begin{gathered}
\left(-i \omega+i k U_{0}\right) a+i k A_{0} v=-\frac{1}{z_{o}} \frac{\partial\left(a U_{0}+v A_{0}\right)}{\partial Z}, \\
\left(-i \omega+i k U_{0}\right) v+3 \mathrm{Oh} k^{2} v-\frac{i k}{2 A_{0}^{3 / 2}}\left(1-k^{2} A_{0}\right) a=-\frac{1}{z_{o}} \frac{\partial\left(v U_{0}\right)}{\partial Z} \\
+\frac{3 i \mathrm{Oh}}{z_{o}}\left(\frac{k}{A_{0}} \frac{\partial A_{0}}{\partial Z} v+2 k \frac{\partial v}{\partial Z}+\frac{\partial k}{\partial Z} v+\frac{k}{A_{0}} \frac{\partial U_{0}}{\partial Z} a\right) \\
-\frac{1}{4 z_{o} A_{0}^{5 / 2}}\left(2 A_{0}\left(1+3 k^{2} A_{0}\right) \frac{\partial a}{\partial Z}+\frac{\partial A_{0}}{\partial Z}\left(3-2 k^{2} A_{0}\right) a+6 k \frac{\partial k}{\partial Z} A_{0}^{2} a\right) .
\end{gathered}
$$

These equations give at leading order $(2.16 \mathrm{a}, \mathrm{b})$ from which we can deduce the dispersion relation (2.17) that defines $k(Z)$. If we now replace $v$ in the right hand side of (B 2a) by its leading order expression in term of $a$, we obtain an expression for $v$ valid up to $O\left(1 / z_{o}^{2}\right)$ terms:

$$
v=\frac{1}{k A_{0}}\left(\left(\omega-k U_{0}\right) a-\frac{1}{z_{o}} \frac{\partial(\omega a / k)}{\partial Z}\right) .
$$

Plugging this expression in (B 2b) with $U_{0}=Q / A_{0}$, we obtain the following equation for $a(z)$

with

$$
R(Z) \frac{\partial a}{\partial Z}+\left(S(Z) \frac{\partial A_{0}}{\partial Z}+T(Z) \frac{\partial k}{\partial Z}\right) a=0
$$

$$
\begin{aligned}
& R(Z)=-2 A_{0} k^{3}+6 A_{0}^{2} k^{5}+4 A_{0}^{3 / 2} k\left(\omega^{2}-3 \mathrm{Oh} i k^{2} \omega+\frac{Q k^{2}\left(-Q+6 i \mathrm{Oh} A_{0} k^{3}\right)}{A_{0}^{2}}\right), \\
& S(Z)=\frac{-8 Q \omega k^{2} A_{0}+3 k^{2}\left(4 Q^{2}+A_{0}^{3 / 2}+4 i \mathrm{Oh} \omega A_{0}^{2}\right)-24 i \mathrm{Oh} Q A_{0} k^{4}-2 A_{0}^{5 / 2} k^{5}}{A_{0}^{3 / 2}}, \\
& T(Z)=2 A_{0}^{1 / 2}\left(-2 \omega^{2} A_{0}+6 i \mathrm{Oh} Q k^{3}+3 A_{0}^{3 / 2} k^{4}\right) .
\end{aligned}
$$

This equation is valid for both downward and upward propagating waves.

For large $Q$, it can be simplified for the downward propagating wavenumbers using $\omega=\bar{\omega} Q$ and $k \sim \bar{\omega} A_{0}+k_{1} / Q$ as

$$
\left(-2 k_{1} A_{0}+3 i \bar{\omega}^{2} \mathrm{Oh} A_{0}^{4}\right) \frac{\partial a}{\partial Z}+\left(5 k_{1} \frac{\partial A_{0}}{\partial Z}-A_{0} \frac{\partial k_{1}}{\partial Z}\right) a=0 .
$$

where $k_{1}(Z)$ is given by $(2.20)$. The amplitude $v(Z)$ is then deduced from $a(Z)$ using (B 3) at leading order in $Q$ :

$$
v(Z)=-\frac{Q}{\bar{\omega} A_{0}^{3}} k_{1} a(Z) .
$$

The two downward propagating waves possess different expressions for $k_{1}$, and thus different amplitudes $a$ and $v$. This guarantees that a combination of the two downward 
propagating waves can be formed such that at the orifice $a=1$ and $v=0$ or $a=0$ and $v=1$.

In the inviscid regime $(\mathrm{Oh} \ll 1)$, equation $(\mathrm{B} 6)$ can be integrated explicitly for any $A_{0}$ as

$$
a^{(i)}(z)=C \frac{A_{0}^{5 / 2}(z)}{\sqrt{k_{1}(z)}},
$$

where $C$ is a constant. It is interesting to compare this expression to the expression $a \sim A_{0}$ that would have been obtained by the argument of Tomotika (1936), that is by

considering the solution as a uniformly stretched fluid cylinder (see Eggers \& Villermaux 2008).

\section{REFERENCES}

Bender, C. M. \& Orszag, S. A. 1978 Advanced mathematical methods for scientists and engineers. New York: McGraw-Hill.

Bers, A. 1983 Space-time evolution of plasma instabilities-absolute and convective, , vol. 1, chap. 3.2. North-Holland.

Chandrasekhar, S. 1961 Hydrodynamic and hydromagnetic stability. Oxford: Clarendon Press.

Clarke, N. S. 1969 The asymptotic effects of surface tension and viscosity on an axiallysymmetric free jet of liquid under gravity. Quart. J. Mech. and Appl. Math 22, 247-256.

Eggers, J. \& Villermaux, E. 2008 Physics of fluid jets. Rep. Prog. Phy 71, 1-79.

FrANkEL, I. \& Weins, D. 1985 Stability of a capillary jet with linearly increasing axial velocity (with application to shaped charges). J. Fluid Mech. 155, 289-307.

Frankel, I. \& WeiHs, D. 1987 Influence of viscosity on the capillary instability of a stretching jet. J. Fluid Mech. 185, 361-383.

Huerre, P. \& Monkewitz, P. A. 1990 Local and global instabilities in spatially developing flows. Annu. Rev. Fluid Mech. 22, 473-537.

Javadi, A., Eggers, Jens, Bonn, D., Habibi, M. \& Ribe, N. M. 2013 Delayed capillary breakup of falling viscous jets. Phys. Rev. Lett. 110 (14), 144501.

LE Dizès, S. 1997 Global modes in falling capillary jets. Eur. J. Mech. B/ Fluids 16 (6), $761-778$

Rubio-Rubio, M., Sevilla, A. \& Gordillo, J. M. 2013 On the thinnest steady threads obtained by gravitational stretching of capillary jets. J. Fluid Mech. 729, 471-483.

Sauter, U. S. \& BugGisch, H. W. 2005 Stability of initially slow viscous jets driven by gravity. J. Fluid Mech. 533, 237-257.

Schlichting, H. 1987 Boundary Layer Theory. McGraw-Hill, Inc., New York.

Schmid, P. J. 2007 Nonmodal stability theory. Annu. Rev. Fluid Mech. 39, 129-162.

Senchenko, S. \& Bohr, T. 2005 Shape and stability of a viscous thread. Phys. Rev. E 71 (056301).

TомотіKA, S. 1936 Breaking up of a drop of viscous liquid immersed in another viscous fluid which is extending at a uniform rate. Proc. R. Soc. London. A 153 (879), 302-318.

Trouton, F., T. 1906 On the coefficient of viscous traction and its relation to that of viscosity. Proc. R. Soc. Lond. 77, 426-440.

VILlERmaux, E. 2012 The formation of filamentary structures from molten silicates: Pele's hair, angel hair, and blown clinker. C. R. Mécanique 340, 555-564.

Weber, C. 1931 Zum zerfall eines flüssigkeitsstrahles. Z. angew. Math. U. Mech. 2, 136. 